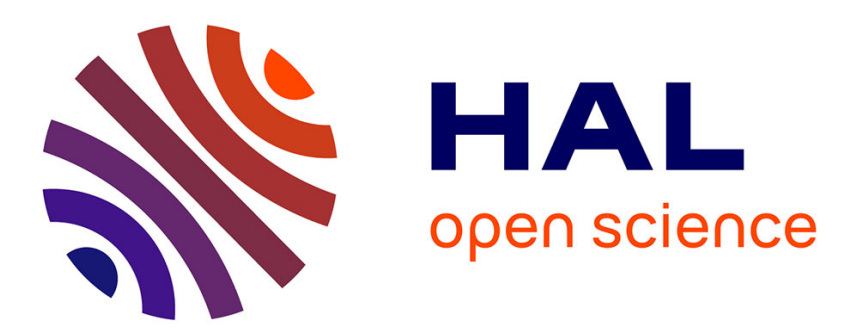

\title{
Numerical simulation of a rotating detonation with a realistic injector designed for separate supply of gaseous hydrogen and oxygen
}

Thomas Gaillard, Dmitry Davidenko, Francis Dupoirieux

\section{- To cite this version:}

Thomas Gaillard, Dmitry Davidenko, Francis Dupoirieux. Numerical simulation of a rotating detonation with a realistic injector designed for separate supply of gaseous hydrogen and oxygen. 2017. hal-01558902

\author{
HAL Id: hal-01558902 \\ https://hal.science/hal-01558902
}

Preprint submitted on $10 \mathrm{Jul} 2017$

HAL is a multi-disciplinary open access archive for the deposit and dissemination of scientific research documents, whether they are published or not. The documents may come from teaching and research institutions in France or abroad, or from public or private research centers.
L'archive ouverte pluridisciplinaire HAL, est destinée au dépôt et à la diffusion de documents scientifiques de niveau recherche, publiés ou non, émanant des établissements d'enseignement et de recherche français ou étrangers, des laboratoires publics ou privés. 


\title{
Numerical simulation of a rotating detonation with a realistic injector designed for separate supply of gaseous hydrogen and oxygen
}

\author{
T. Gaillard*, D. Davidenko, F. Dupoirieux \\ ONERA, F-91120 Palaiseau
}

\begin{abstract}
This paper presents the numerical results for a Rotating Detonation (RD) propagating in a layer of combustible mixture, created by injection of gaseous hydrogen and oxygen. 3D Large Eddy Simulations (LES) of a reacting flow have been performed in a domain of planar geometry in order to eliminate possible effects of the chamber curvature. First, the results for a 2D case with uniformly distributed premixed injection are presented to characterize the RD propagation under the most idealized conditions. Then a 3D concept is introduced for the injector composed of injection elements, whose operation in the established regime was previously studied by the authors. The RD propagation is simulated under the conditions of premixed and separate injection of the propellants at globally stoichiometric proportions. The case of separate propellant injection is the most realistic one. The computational results, represented by instantaneous and averaged flowfields, are analyzed to point out the changes in the conditions of RD propagation induced by the injection through discrete holes with respect to the distributed one and by the switching between the premixed and separated modes of injection. Macroscopic quantities, such as the detonation propagation speed, mean chamber pressure, average parameters of the mixture, and mixture efficiency are evaluated and compared in order to characterize the studied effects.
\end{abstract}

*Corresponding author, Email: thomas.gaillard@onera.fr 
Keywords: Rocket propulsion, rotating detonation, Large Eddy Simulation (LES), propellant injection

\section{Introduction}

The interest in the rotating detonation $(\mathrm{RD})$ to enhance rocket propulsion efficiency was already noticeable in the 1960s 1, 2, 3. However the research activity in that field has strengthened in the last two decades with the possibility to perform complex unsteady numerical simulations which complement the experimental work achieved to evaluate the feasibility and expected gain of Rotating Detonation Engine (RDE) concepts. The issues that must be tackled to bring these concepts to reality are summarized in different papers such as [4, 5, 6. A large amount of experimental work has been carried out with the objective to clearly identify these issues and prepare technological solutions. Different types of fuel and fuel-oxidizer mixtures have been tested, see for example [7, 8, 9, 10, 11]. In a first step, the rotating detonation is often studied in small combustion chambers but demonstration chambers are now in development or testing [12. Semi-analytical and simple 0D models have been

15 developed to evaluate the theoretical performance of RDE, as in [13, 14]. Even if parametric studies are more complex with $2 \mathrm{D} / 3 \mathrm{D}$ numerical tools, numerical simulations are now commonly used, as in [15, 16, 17, 18, 19, 14, 20, 21, because they can depart from too simplifying assumptions and give a deep insight into the flow physics. Most of time the injection of a perfect fuel-oxidizer mixture was considered in the simulations.

Although the preparation of a homogeneous fuel-oxidizer mixture upstream of the combustion chamber is technically possible and helpful to ensure correct propagation of the RD, the premix injection can be troublesome: a deflagration initiated by the contact of fresh gases with hot gases, when the injection 25 is blocked after the RD passage, may propagate upstream in the injector. Another risk is the detonation transmission through the injection holes. To avoid these two risks, one should respect some limitations related to the hole diam- 
eter. In case of deflagration, reducing the diameter provides an increase in thermal losses at walls, which leads to deflagration quenching. For detonation transmission, there also exists a critical diameter determined from the equality between the hole perimeter and the detonation cell size. Below this particular diameter, detonation cannot propagate in the injection tubes. For example, for a stoichiometric hydrogen-oxygen mixture at a pressure of $1 \mathrm{MPa}$, the critical diameter is about $20 \mu \mathrm{m}$. Such a severe limitation would lead to important technological issues as well as high pressure losses. In fact, the only way to fully prevent combustion from propagating inside the injector is to feed the oxidizer and fuel separately as in the practice of RD experiments. However, the necessity to mix the fresh propellants only inside the combustion chamber results in less favorable conditions for the RD propagation. For example, a propagation 40 speed deficit amounting up to $20 \%$ with respect to the ideal Chapman-Jouguet (CJ) detonation under premixed conditions has been found in the experiment described in 8 .

An evolution from a very idealistic to more realistic approach in simulation of propellant injection can be observed during the last decade. For example, in 45 first 2D simulations, Zhdan [15] and Davidenko et al. 22] considered uniformly distributed injection. Then, to account for the section variation from the injector to the chamber, slotted injection was used by Eude et al. [23]. Five different slotted injection patterns were simulated and compared by Liu et al. [24]. Schwer et al. 25] analyzed the pressure feedback in the injector due to the detonation propagation over a series of holes. However, in the aforementioned simulations the injected propellants were perfectly mixed. The work of Frolov et al. [26] is pionneering in the sense that a 3D simulation of a $\mathrm{RD}$ with a real geometry and separate injection of gaseous propellants could be carried out. In [27, separate injection of $\mathrm{H}_{2}$ and air is considered but only a cold flow simulation is presented in parallel with an extension of the "Inductiontime Parameter Model" for a $\mathrm{H}_{2} /$ air detonation in non-stoichiometric mixtures. Separate injection is also considered in 28 but the simulation is nonreactive and the computational domain is reduced to a sector of an RDE annulus. Recently, 
Cocks et al. 29] have also achieved 3D calculations of rotating detonations with separate injection in a configuration studied at the Air Force Research Laboratory.

The authors of the present paper proposed in [30] a realistic manufacturable configuration for separate injection of gaseous hydrogen and oxygen, which was optimized using nonreactive unsteady 3D simulations. Considering the small number of contributions concerning the $3 \mathrm{D}$ simulation of $\mathrm{RD}$ with separate injection of fuel and oxidizer, we present here, as an extention of our previous work, simulation results on the propagation of a RD over a row of optimised injection elements from [30.

In section 2, the context of the study linked to the operation of a RDE 70 is presented. In section 3 , the computationnal approach is explained. Some preliminary simulations of a detonation are run with the CEDRE code. Then, section 4 presents a first series of $2 \mathrm{D}$ reference results on the RD propagation with uniformly distributed premixed injection. Section 5 reminds the characteristics of the optimised injection configuration previously studied and used in the

$753 \mathrm{D}$ computations of a RD. Section 6 is dedicated to the numerical methodology of the 3D LES simulations, with a large number of injection elements arranged in series. Section 7 gives additional details on the averaging procedures used to post-process the 3D simulation results. The main features of the reactive flow are finally presented in section 8 for an established RD propagation. The effect of premixed and separate injection regimes are compared through the evaluation of the RD propagation speed, the chamber pressure and the average mixture conditions obtained in front of the RD. The conclusion section summarizes the obtained numerical results and suggests improvements of the injector optimization.

\section{2. RDE operation principle}

Among possible configurations of RDE combustion chambers, the one with an annular cylindrical combustion chamber is considered in the present study. 
Its operation principle is schematically shown in Fig 1 . The fuel and oxidizer (1) are fed through holes in the injection wall (2). After detonation initiation 90 at the engine start, one or several detonation fronts (3) propagate in the layer of combustible mixture (4) created by the propellant injection. The height of the detonation front, $h$, and the spatial period, $l$, between successive fronts are proportional and depend on the propellants and injection conditions. At a stable operation regime, the detonation waves propagate continuously in the same azimuthal direction thus having rotational motion about the chamber axis. The detonation waves induce oblique shocks (5) in the burnt gases. Combustion products generated by the detonation waves expand in the chamber and discharge through the open end (6) of the duct.

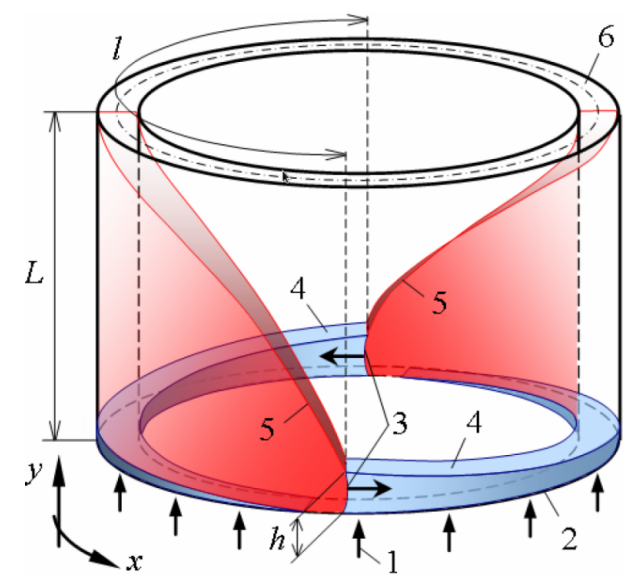

Figure 1: Principle of the RDE operation: 1 - propellant injection; 2 - injection wall; 3 detonation fronts; 4 - fresh mixture layer; 5 - oblique shocks; 6 - outlet section; $h$ - height of detonation front; $l$ - spatial period between successive detonation fronts; $L$ - chamber length.

\section{Computational approach for RD simulation}

Although the present paper is devoted to simulation and analysis of $\mathrm{RD}$ propagation under realistic injection conditions, the chamber is considered in a very simple way with no relation to a particular geometry of the duct. The main 
reason for this is the intention to study the effects due to the injection without coupling them with the other ones, such as the effects due to the curvature of the annular passage and the viscous interaction on the cylindrical walls. It was shown by Eude et al. 31] that the 3D flowfield in an annular chamber becomes close to the $2 \mathrm{D}$ flowfield if the mean radius of the annulus is sufficiently large with respect to the duct width (radial distance between the cylindrical walls), hence there will be no critical change in injector operation if used in a real chamber. Another reason for duct geometry simplification is to limit the computational cost of $3 \mathrm{D}$ simulations thus allowing simulations with different conditions for comparative analysis and during sufficiently long physical time to obtain well established flowfields.

The simplest computational domain can be obtained by cutting and unrolling the annular duct to obtain a planar one with periodicity conditions on the cutting surfaces. By assuming equidistant detonation fronts propagating at a constant speed, it is possible to consider a computational domain covering only one period $l$ as shown in Fig,2, The computational domain has two additional parts: i) an injector zone of length $L_{j}$, which can be represented by a slot (Fig 2 or a series of injection holes (Fig 7) with prescribed inlet conditions; ii) a divergent duct of length $L_{d}$ to obtain supersonic outflow and make the simulated flow independent of the downstream conditions.

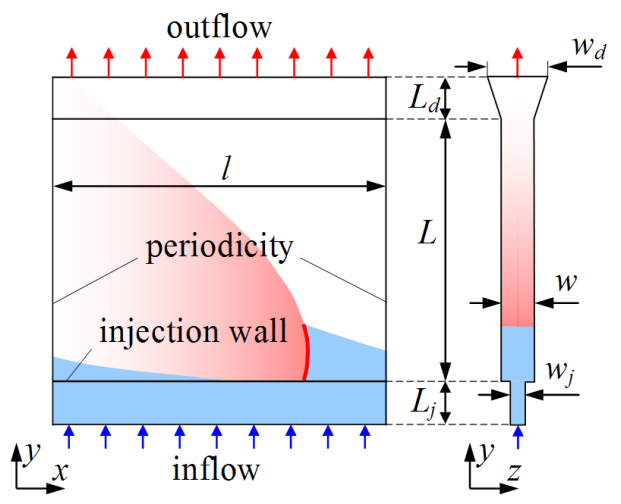

Figure 2: Schematic of the computational domain for RD simulation. 
The detonation simulations considered in this paper were performed on a small computational domain with the following dimensions: $l=50 \mathrm{~mm}, L=$ $20 \mathrm{~mm}$. The chosen period $l$ is a lower estimation based on the approximate relations, proposed by Bykovskii et al. [7, for a detonation cell size on the order of $1 \mathrm{~mm}$. The value of $L$ is large enough for detonation propagation unaffected by the duct divergence; it is confirmed both experimentally [7] and numerically [22] that $L=2 h$ is sufficient.

The injection conditions are defined as follows: the total temperature of both propellants is $300 \mathrm{~K}$; the global equivalence ratio is 1 ; the total mass flow rate per unit area of the chamber cross section is $100 \mathrm{~kg} /\left(\mathrm{s} \cdot \mathrm{m}^{2}\right)$. Such conditions are relevant to ground testing of a model RDE.

All computational results presented in this paper were obtained using the CEDRE code [32, 33, which is a CFD software developed at ONERA for numerical simulations in application to energetics and propulsion. CEDRE integrates several numerical solvers based on a finite-volume method for general unstructured meshes. In the frame of the present study, Navier-Stokes equations were solved to simulate a reactive flow of multispecies compressible gas. Flow turbulence is simulated using the LES approach with the Smagorinsky subgrid viscosity model. Convective fluxes at the faces of the mesh cells are determined using the classical HLLC method of Riemann problem solution. Second-order accuracy in space is obtained due to a MUSCL scheme with the Van Leer slope limiter for the convective fluxes and the central-difference scheme for the viscous fluxes. Time integration is performed using an accurate implicit scheme with the GMRES method for solving the linearised equation system.

The reactive gas is represented by a mixture of 6 chemical species $\left(\mathrm{H}_{2}, \mathrm{O}_{2}\right.$, $\left.\mathrm{H}_{2} \mathrm{O}, \mathrm{H}, \mathrm{O}, \mathrm{OH}\right)$ treated as ideal gases with standard temperature-dependent properties. The chemical kinetic model includes 7 reversible reactions. This model was tested by Davidenko et al. 222 and found sufficiently accurate for detonation simulation with respect to more complex mechanisms. Molecular transport properties of the species are defined by constant Prandtl and Schmidt numbers. As the present study is focused on the mixing process between the 
fresh propellants, the transport coefficients of $\mathrm{H}_{2}$ and $\mathrm{O}_{2}$ were determined for their mixture at stoichiometric proportions. For the other species, pure species properties are used.

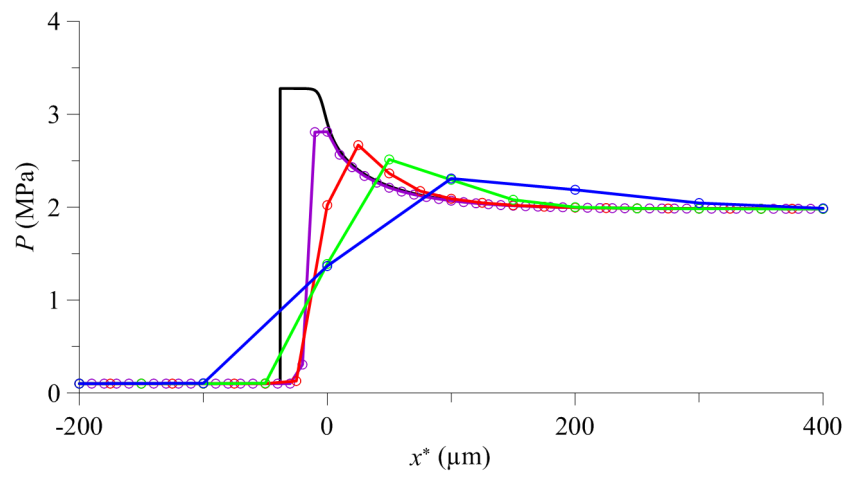

a)

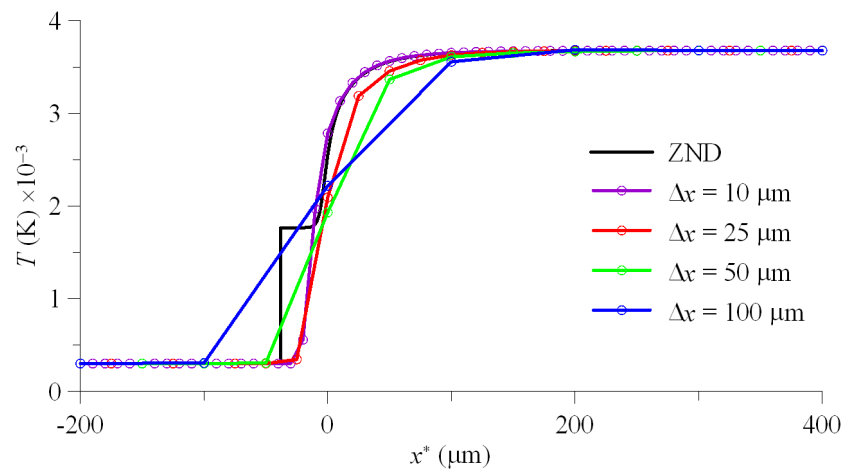

b)

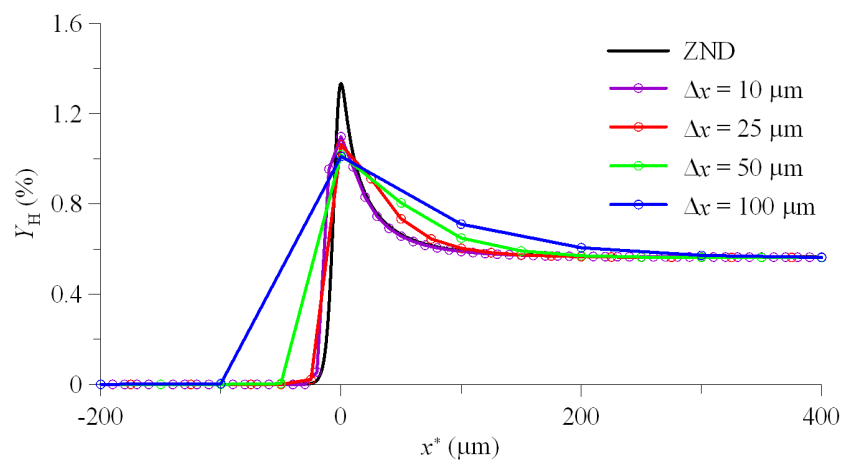

Figure 3: Results for a CJ detonation in a $\mathrm{H}_{2}-\mathrm{O}_{2}$ stoichiometric mixture at $0.1 \mathrm{MPa}$ and $300 \mathrm{~K}$ from a ZND model (reference solution) and CEDRE simulations with different grid steps $\Delta x$ : a) static pressure; b) static temperature; c) mass fraction of the $\mathrm{H}$ radical. Coordinate transformation: $x^{*}=x-x\left(Y_{\mathrm{H}, \max }\right)$. 
In the $\mathrm{RD}$ simulations presented below, the chemical kinetic model was active in the part of the computational domain above the injection wall, whereas in the injector zone, the gas mixture was treated as non-reactive. This was necessary in the case of premixed injection in order to prevent detonation propagation inside the injector. It should be stressed that the premixed injection is considered here with the only purpose to identify the flowfield changes between the premixed and separate injection modes and not as a real operation mode.

Before performing RD simulations, several 1D test cases were run to define the numerical resolution necessary for correct simulation. Precise simulation of the inner structure of a detonation wave in a $\mathrm{H}_{2}-\mathrm{O}_{2}$ stoichiometric mixture requires spatial resolution on the order of $1 \mu \mathrm{m}$, which leads to a prohibitively large mesh size. Most of known RDE simulations were performed with relatively coarse resolution sufficient for correct prediction of the detonation speed and overall change of flow parameters, which is appropriate for stable propagation regimes. In Fig 3 , the results of 1D simulations by CEDRE are compared with the Zeldovich-von Neumann-Döring (ZND) solution for a Chapman-Jouguet (CJ) detonation propagating in a $\mathrm{H}_{2}-\mathrm{O}_{2}$ stoichiometric mixture with the initial conditions $0.1 \mathrm{MPa}$ and $300 \mathrm{~K}$. The ZND profiles of pressure and temperature show that combustion starts at about $30 \mu \mathrm{m}$ behind the shock front. In the CEDRE simulations with different grid steps $\Delta x=10,25,50$ and $100 \mu \mathrm{m}$, the induction zone behind the shock front is not resolved but even with the coarsest grid, the final CJ state is correctly predicted; it is also verified that the detonation speed corresponds to the theoretical value $2836 \mathrm{~m} / \mathrm{s}$. The grid step $\Delta x=100 \mu \mathrm{m}$ is the maximum allowable for detonation simulation with initial conditions close to the chosen ones because the use of larger grid steps leads to an important deficit in detonation speed.

No particular model of combustion-turbulence interaction is used to treat the deflagration front at the contact surface between the fresh mixture and the 185 burnt gases. To see to what extent the prediction of the deflagration speed will be correct with the chosen gas model and grid resolution, 1D laminar flame simulations were performed. Although the deflagration front may be subject to 
complex 3D perturbations, these 1D simulations can give a first order prediction of the deflagration speed. Freely propagating flames in a $\mathrm{H}_{2}-\mathrm{O}_{2}$ stoichiometric mixture with an initial temperature of $300 \mathrm{~K}$ were simulated at different pressures within the range corresponding to the variation along the fresh mixture layer in the RD simulations presented below. Reference results were obtained by the PREMIX code from CHEMKIN-II with a recently published chemical kinetic mechanism from the University of San Diego 34, which was validated for $\mathrm{H}_{2}$ /air premixed laminar flames [35]. A finely adapted grid and a detailed modeling of molecular transport were used in the PREMIX simulations to obtain accurate solutions. The CEDRE simulations of the flame propagation result in a stronger pressure dependence with respect to the PREMIX results due to the simplified modelling approach. As a summary of this comparative study, the deflagration speed predicted by CEDRE is overestimated by $27 \%$ at $p=$ $2 \mathrm{MPa}$ and underestimated by $24 \%$ at $p=1 \mathrm{MPa}$. Nevertheless, the error in the laminar flame speed is not crucial in the RD simulations because the rate of fresh mixture consumption by the deflagration is typically an order of magnitude smaller than that by the detonation.

\section{4. $R D$ in quasi-2D configuration with premixed injection}

The present case is characterised by continuously distributed and fully premixed injection, which provides the most idealized conditions of the fresh mixture in the combustion chamber. The corresponding simulation results will be used as reference for comparison with the $3 \mathrm{D}$ results presented later for the injector with discrete holes. The geometrical configuration of this case is schematically shown in Fig 2 In addition to the $l$ and $L$ parameters already specified, the others are $L_{j}=L_{d}=2 \mathrm{~mm}, w_{j} / w=0.2$ and $w_{d} / w=3$. The absolute value of $w$ is unimportant because this configuration is treated as quasi-2D, which means that in spite of the duct section variation in the $z$ direction, the flow is considered uniform in this direction as the computational mesh has a single layer of cells between the opposite walls. Uniform boundary conditions 
of constant mass flux are imposed at the inflow boundary of the slot injector. This technique allows simulating a flow in the chamber very close to the one obtained in 2D simulations with similar conditions [31.

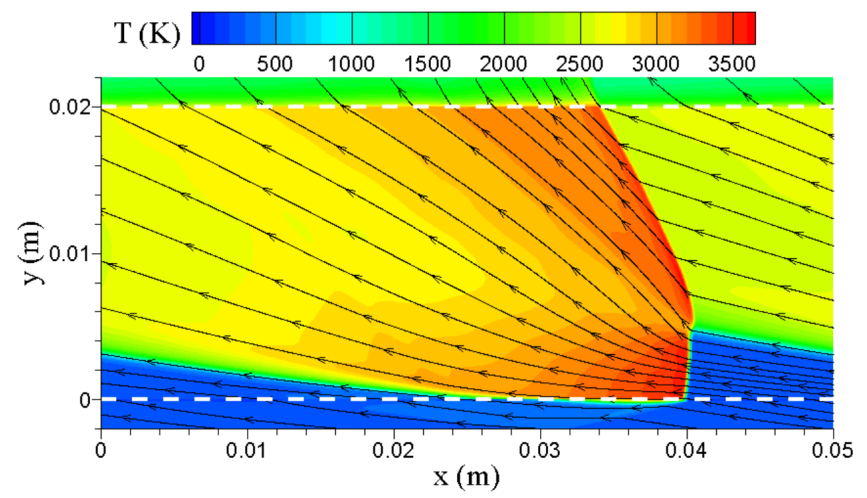

Figure 4: $\mathrm{RD}$ in quasi-2D configuration with premixed injection: Instantaneous temperature field with superimposed streamlines in the moving reference frame attached to the detonation front. White dashed lines delimit the chamber domain.

An instantaneous flowfield, corresponding to fully established RD, is illustrated in Fig.4. In the temperature field, one can easily recognize the flow structure by referring to the schematic representation in Fig 1 For more complete illustration, streamlines are traced in the moving reference frame attached to the detonation front, in which the flowfield is in steady state. The detonation front is almost normal to the incoming flow of fresh mixture; the following burnt gas expansion is clearly identified by the streamline divergence. On a distance of approximately $14 \mathrm{~mm}$ behind the detonation wave, high-pressure gases block propellant injection and even penetrate a little inside the injector. The following pressure decrease along the injector wall allows fresh mixture reinjection in the chamber. The oblique shock, induced by the interaction between the expanding high-pressure gases behind the present detonation and the low-pressure flow from the previous detonation, is identified by the sharp increase of temperature and the turn of streamlines. The flowfield is smooth due to the relatively coarse mesh resolution, which inhibits instabilities of the detonation front and 
characterize the obtained flowfield as idealized.

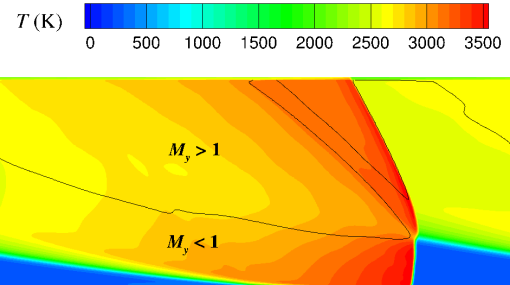

a)
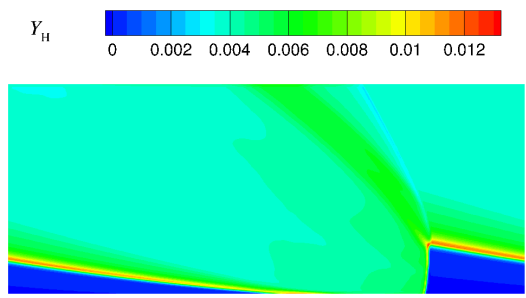

c)

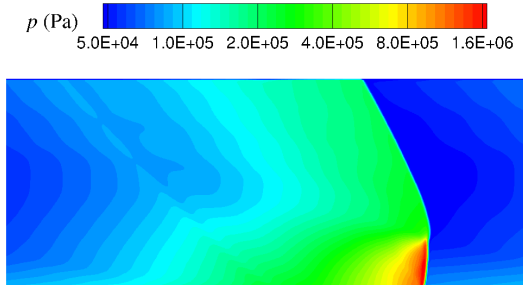

b)
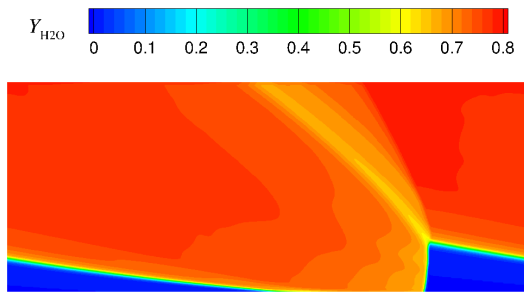

d)

Figure 5: Instantaneous flowfields from quasi-2D simulation: a) static temperature with the $M_{y}=1$ contour; b) static pressure; c) H-radical mass fraction; d) $\mathrm{H}_{2} \mathrm{O}$ mass fraction.

More flowfields are shown in Fig 5 for the chamber region $(y \in[0,20] \mathrm{mm})$. The temperature field is shown once again with the $M_{y}=1$ contour, above which the $y$-component of the flow velocity is supersonic. This contour delimits a wake-like narrow zone behind the oblique shock corresponding to the layer of combustion products from deflagration. The static pressure field shows a smooth pressure variation in a wide range from the highest level behind the detonation front to the lowest one in front of the oblique shock. In the H-radical field, the deflagration is marked by the maximum level of $\mathrm{H}$ mass fraction. In the detonation front, the $\mathrm{H}$ peak is narrower and truncated by the coarse mesh resolution as illustrated in Fig 3 c. H radicals remain at a certain concentration in the combustion products due to their partial dissociation, which follows the temperature evolution. By comparing the fields of temperature and $\mathrm{H}_{2} \mathrm{O}$ mass fraction, one can observe progressive formation of $\mathrm{H}_{2} \mathrm{O}$ from radical re- 
following $\mathrm{H}_{2} \mathrm{O}$ dissociation when the combustion products are recompressed by the oblique shock.

The time history of the wall pressure at a particular $x$ position is shown in Fig 6 for a short time period after the RD establishment. Considering the 255 $\pm 3 \mathrm{~m} / \mathrm{s}$.

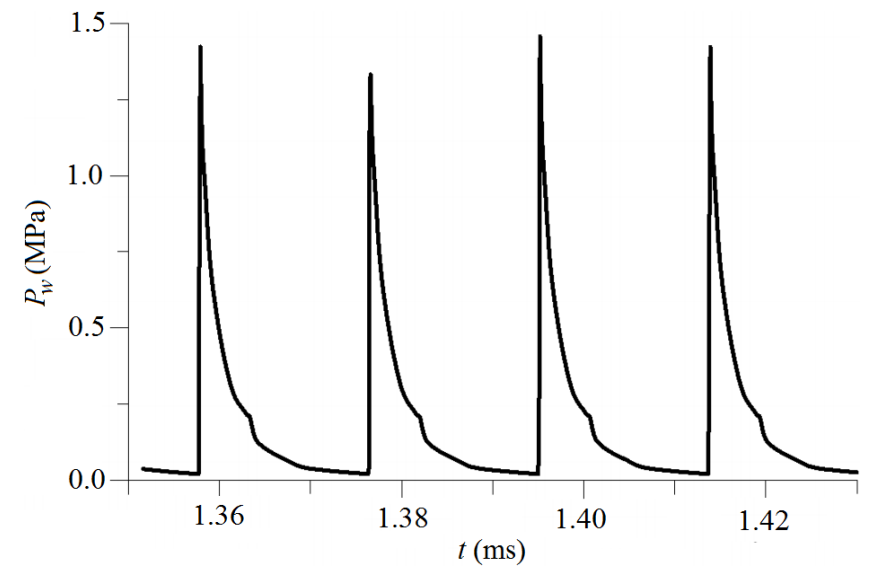

Figure 6: RD in quasi-2D configuration with premixed injection: Time history of the wall pressure at a particular $x$ position.

The fresh mixture layer is analysed in a vertical cross section close to the detonation front. The layer height $h=4.95 \mathrm{~mm}$ is determined as the vertical distance between the injection wall and the deflagration front ( $\mathrm{H}$ radical peak). By integrating the mass flux in the moving reference frame attached to the detonation front within the range $y \in[0, h]$, the mass flow rate of fresh mixture burnt by the detonation is about $90 \%$ of the injected mass flow rate; the 
$10 \%$ loss is due to the deflagration. The following mean flow conditions are determined within the range $y \in[0,4] \mathrm{mm}$ : static pressure $62.8 \mathrm{kPa}$; static temperature $238 \mathrm{~K}$; velocity $x$-component $-35 \mathrm{~m} / \mathrm{s}$; Mach number from the velocity $y$-component 0.75 . The detonation velocity with respect to the fresh mixture flow is approximately $2714 \mathrm{~m} / \mathrm{s}$, which is about $4 \%$ lower than the Chapman-Jouguet detonation velocity determined for the same pressure and temperature.

Considering the flowfield in a horizontal plane at $y=19.5 \mathrm{~mm}$, it is found that the flow is supersonic everywhere with the Mach number ranging from 1.1 to 1.42. The velocity $y$-component is supersonic in the major part of almost $80 \%$ of this section; it becomes fully supersonic at $y \approx 20 \mathrm{~mm}$.

\section{Injection configuration for the $3 \mathrm{D}$ simulations}

The injection configuration considered here for the $3 \mathrm{D}$ simulations of $\mathrm{RD}$ is the one selected in [30]. It is a periodic pattern in both coordinate directions of unlike semi-impiging jets, which provides better mixing efficiency than purely impinging or sheared configurations, on the one hand, and than patterns with symmetric pairs of injection elements, on the other hand. Figure 7 shows two neighbouring injection elements in a row along the $x$-axis in order to illustrate their layout on the injector face (2) in Fig,1. The angle of the feeding pipes with the direction normal to the injector face is equal to $30^{\circ}$ and the angle $\alpha$ between the two jets is $60^{\circ}$. The elliptical sections of the injection holes have parallel major axes. The angle $\beta$ between the line passing through the centres of the ellipses and their major axes is equal to $45^{\circ}$. In order to avoid intersection of the feeding pipes from neighbouring injection elements, each pair of pipes is set at the angle $\gamma$ equal to $13^{\circ}$ with respect to the $x$-axis as shown in Fig.7.

In the RD simulations presented below, 21 injection elements are set in a single row along the $x$-axis by the same way as illustrated in Fig, 7 Each element occupies a square on the injector face and the total area of round injection holes represents $20 \%$ of the injector face area. The diameters of $\mathrm{H}_{2}$ and $\mathrm{O}_{2}$ holes are 


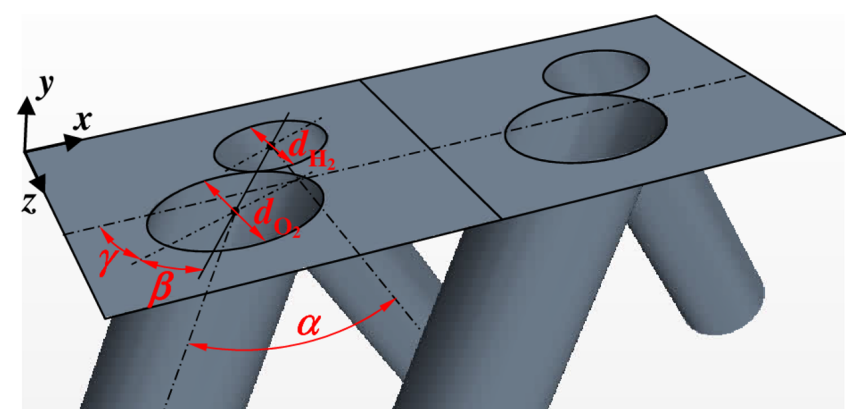

Figure 7: 3D view of two injection elements in a row.

$d_{\mathrm{H}_{2}}=0.71 \mathrm{~mm}$ and $d_{\mathrm{O}_{2}}=1 \mathrm{~mm}$ respectively, which is less by a factor $\sqrt{2}$ than in the configuration studied in [30]. This geometrical reduction is necessary to comply with the small size of the computational domain for RD simulation. Smaller injection elements will normally improve propellant mixing close to the injector face. The element dimensions along the $x$ and $z$-axis are respectively $2.45 \mathrm{~mm}$ and $2.4 \mathrm{~mm}$.

$3 \mathrm{D}$ simulations of $\mathrm{RD}$ were carried out with the given injection configuration. Some specific cases of separate propellant injection were also simulated 305 by varying the diameter of $\mathrm{O}_{2}$ holes, $d_{\mathrm{O}_{2}}$, in order to study its influence on the propellant mixing. These results are briefly described in section 8

\section{Numerical methodology for the $3 \mathrm{D}$ computations}

Contrary to the $2 \mathrm{D}$ case presented above, $3 \mathrm{D}$ computations show much more perturbations in the flowfield due to the following factors: i) because of hydrodynamic instabilities, the mixing process itself produces highly turbulent flow close to the injector face as shown by the detailed study on a single injection element [30; ii) imperfect mixing of the injected propellants and presence of burnt gases between the propellant jets result in a strong intermittency in terms of chemical composition and temperature in the fresh mixture layer, which perturbs the detonation front, thus resulting in a strongly nonuniform flow of combustion products behind the detonation; iii) because of the short time period between 
successive passages of detonation fronts, acoustic perturbations generated by a given detonation will remain in the chamber and interact with the next one, thus contributing to the overall level of flow instabilities [23]. To simulate these which are cut or deformed at the domain boundaries to be conformal with their shape. Two computational meshes were used in the simulations: a coarse one with $100 \mu \mathrm{m}$ cells and a fine one with $50 \mu \mathrm{m}$ cells. The indicated cell size was used for the injector subdomain and the lower part of the chamber subdomain $345(y \leq 12.8 \mathrm{~mm})$. In the upper part of the chamber subdomain $(y \geq 12.8 \mathrm{~mm})$, less important for the simulation, the mesh was progressively coarsened up to a cell size of $400 \mu \mathrm{m}$. The total numbers of cells for coarse and refined meshes 
are 1.8 and 14 million respectively. Figure 8 indicates the different boundaries numbered from 1 to 7 . For the propellant inlet boundaries (1), constant mass flux, total temperature and gas composition are imposed together with a nonreflective condition to absorb the shocks coming from the chamber when the detonation passes over the injection holes. Inviscid flow conditions are used for the boundaries representing the walls of the injector feeding pipes (2), the injector face wall (3) as well as the divergent duct (6). Periodicity conditions are applied to the opposite boundaries of the chamber subdomain (4) and (5). Finally, supersonic outflow conditions are imposed on the outlet boundary (7).

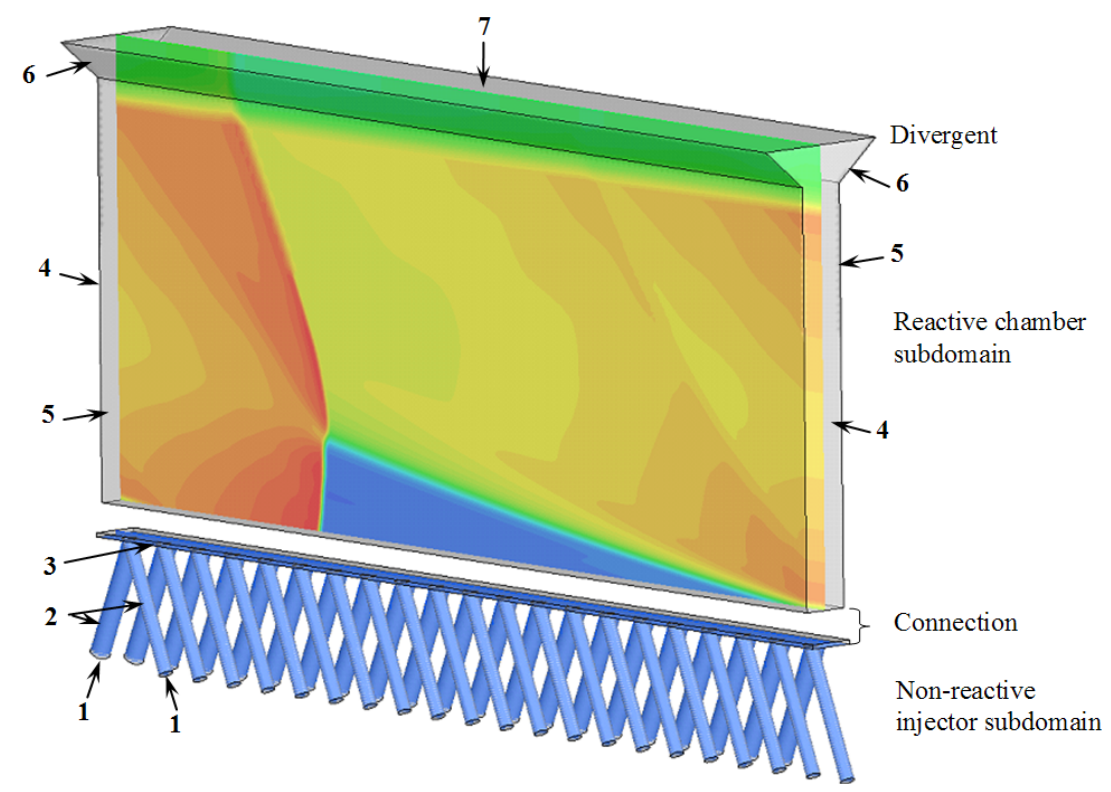

Figure 8: Computational domain split into the injector and chamber subdomains with schematic representation of the initial conditions. Boundaries types: 1 propellant inlet; 2 walls of the feeding pipes; 3 injector face wall; 4 periodic boundaries; 5 chamber walls; 6 divergent duct walls; 7 outlet.

According to the strategy adopted for the present study, several cases were studied. These cases are identified in Tab.1. In the case designation, the $1^{\text {st }}$ digit represents the number of dimensions; the letter in second place identifies the mesh resolution ("C" stands for coarse, "F" for fine); the letter in third 
place defines the injection mode ("P" for premixed, "S" for separate). Case $2 \mathrm{CP}$ corresponds to the $2 \mathrm{D}$ simulation presented above whereas the other cases are $3 \mathrm{D}$. Case $3 \mathrm{CP}$ is first simulated in order to study the effect of the $3 \mathrm{D}$ injector with discrete holes while keeping the same mesh resolution in the $x y$ plane and injected composition as in Case 2CP. Then Case 3CS is simulated by changing the injection mode from premixed to fully separate. Finally Case 3FS is considered to investigate the effect of the mesh resolution on the simulated flowfield.

\begin{tabular}{c|c|c|c} 
& \multicolumn{3}{|c}{ Table 1: Studied cases of RD simulation. } \\
Case & Dimension & Resolution $(\mathbf{\mu m})$ & Propellants \\
\hline \hline 2CP & $2 \mathrm{D}$ & 100 & premixed \\
3CP & $3 \mathrm{D}$ & 100 & premixed \\
3CS & $3 \mathrm{D}$ & 100 & separate \\
$3 \mathrm{FS}$ & 3D & 50 & separate
\end{tabular}

The simulation of Case 3CP is initialised using the following two steps: i) a non-reacting flow of premixed propellants with nominal injection conditions is simulated with a large time step to establish the flowfield in the injector subdomain; ii) the reactive flowfield from Case $2 \mathrm{CP}$ is projected onto the chamber subdomain as initial condition for the following reactive simulation. This technique allows a quick transition to an established flowfield of Case $3 \mathrm{CP}$. It was not possible to start the simulation of Case 3CS directly from the flowfield of Case $3 \mathrm{CP}$ by suddenly changing the injection conditions from the premixed to fully separate mode. Instead, the injected composition was changed by smaller steps allowing a smooth transition of the reactive flowfield. Finally, the simulation of Case 3FS was initiated by interpolating the flowfield of Case 3CS on the fine mesh. 


\section{Averaging of the 3D flowfields}

For the analysis and comparison with the 2D flowfield, the simulated 3D flowfields were averaged both in space and time by using the method described below. For each 3D case, the processed and analysed flowfields correspond to the established flow obtained when the chamber pressure is stabilised at a certain level and the RD propagates at a constant speed.

\subsection{Spatial average}

Spatial averaging is performed on instantaneous flowfields within the injector and chamber subdomains in the $y$-range from 0 to $20 \mathrm{~mm}$. The physical quantities at a given time $t^{n}$ are averaged along the $z$-direction. For pressure, density and some other quantities, simple arithmetic averages are calculated, whereas temperature, velocity components and species mass fractions are mass-averaged by using the following formula:

$$
\widetilde{q}_{i, j}^{n}=\frac{\sum_{k}^{K}(\rho q)_{i, j, k}^{n}}{\sum_{k}^{K} \rho_{i, j, k}^{n}}
$$

where $i, j$ and $k$ are cell indices in the $x, y$ and $z$ directions respectively; $K$ is the number of cells in the $z$-direction; $q$ is the averaged quantity; $\rho$ is the gas density. The cell geometry is not used for averaging because of the Cartesian mesh with a uniform cell size.

\subsection{Time average}

Schwer et al. 25] used time averaging of instantaneous flowfields for describing their results from 3D simulations of $\mathrm{RD}$. As the flowfield is highly unsteady in our simulations, the flowfields were processed in a similar way. Time averaging is performed on spatially-averaged instantaneous flowfields (snapshots) obtained with a fixed sampling frequency. A first step of data processing consists in translating each snapshot along the $x$-axis such that the detonation 
$x_{D}$ for a particular snapshot is determined as the most advanced point along the $x$-axis where pressure exceeds an imposed threshold. The new coordinate after translation is $x^{*}=x-x_{D}+x_{D}^{*}$, to which we apply the following periodic shift:

$$
x_{p e r}^{*}=\left\{\begin{array}{l}
x^{*}+l, \quad x^{*}<0 \\
x^{*}, \quad 0 \leq x^{*} \leq l \\
x^{*}-l, \quad x^{*}>l
\end{array}\right.
$$

From $N$ snapshots, time averaging of any quantity at point $(i, j)$ is performed similarly to the spatial average procedure. Each quantity is averaged using similar principles for the spatial averaging. Mass-averaged quantities are determined by the formula:

$$
<\widetilde{q}_{i, j}>=\frac{\sum_{n}^{N} \widetilde{\rho}_{i, j}^{n} \widetilde{q}_{i, j}^{n}}{\sum_{n}^{N} \widetilde{\rho}_{i, j}^{n}}=\frac{\sum_{n}^{N} \sum_{k}^{K}(\rho q)_{i, j, k}^{n}}{\sum_{n}^{N} \sum_{k}^{K} \rho_{i, j, k}^{n}}, \text { with } \widetilde{\rho}_{i, j}^{n}=\frac{1}{K} \sum_{k}^{K} \rho_{i, j, k}^{n}
$$

\subsection{D data processing for RD characterisation}

For quantitative analysis of the simulation results, it is important to evaluate some integral characteristics of $\mathrm{RD}$ from the $3 \mathrm{D}$ unsteady flowfields. The processed data represent $N$ snapshots of the $3 \mathrm{D}$ flowfield recorded with a fixed time period. The detonation propagation speed $V_{D}$ is determined as a mean of $N-1$ values $V_{D}^{n}=\left(x_{D}^{n+1}-x_{D}^{n}\right) /\left(t^{n+1}-t^{n}\right)$ between each pair of snapshots.

${ }_{420}$ To characterize the fresh mixture layer in front of the detonation wave, a control volume is determined on the following coordinate extents. The $x$-range is defined from the detonation front position plus a small shift $x_{\min }=x_{D}+0.3$ $\mathrm{mm}$, providing no perturbation from the front, to $x_{\max }=x_{\min }+l / N_{\mathrm{inj}}$, where $l / N_{\text {inj }}$ is the size of a single injection element. The $y$-range is defined from the 

$Z_{\mathrm{O}_{2}}$. Now consider that the stoichiometric mixture is burnt by $50 \%$. By using the global reaction expression $2 \mathrm{H}_{2}+\mathrm{O}_{2}=2 \mathrm{H}_{2} \mathrm{O}$ and substituting $\mathrm{BG}$ for $\mathrm{H}_{2} \mathrm{O}$, we find $Z_{\mathrm{H}_{2}}=Z_{\mathrm{O}_{2}}=0.25$ and $Z_{\mathrm{BG}}=0.5$ by definition. The same values for

where $X_{\mathrm{H}_{2}}$ and $X_{\mathrm{O}_{2}}$ are the mole fractions of $\mathrm{H}_{2}$ and $\mathrm{O}_{2}$ respectively; $X_{\mathrm{BG}}$ is the sum of the mole fractions of the other species $\left(\mathrm{H}, \mathrm{O}, \mathrm{OH}, \mathrm{H}_{2} \mathrm{O}\right)$. The $Z$ parameter is designed for proper visualization of components fields because of the following properties. In a stoichiometric mixture without burnt gases $\left(X_{\mathrm{BG}}\right.$ $=0$ ), mole fractions of $\mathrm{H}_{2}$ and $\mathrm{O}_{2}$ satisfy $X_{\mathrm{H}_{2}}=2 X_{\mathrm{O}_{2}}$ so that $Z_{\mathrm{H}_{2}}=Z_{\mathrm{O}_{2}}=0.5$ by definition. This is convenient for graphical representation of the mixing zone indicating the right proportions of propellants by equilibrated values of $Z_{\mathrm{H}_{2}}$ and 
$Z_{\mathrm{H}_{2}}, Z_{\mathrm{O}_{2}}$ and $Z_{\mathrm{BG}}$ are obtained in the case of dilution by the burnt gases by $40 \%$. Therefore, $Z_{\mathrm{BG}}$ represents a convenient parameter to estimate the degree of dilution or burning of the fresh mixture. We can go further by introducing three more parameters defined by the following expressions:

$$
\begin{aligned}
Z_{\mathrm{H}_{2}, \mathrm{ex}} & =Z_{\mathrm{H}_{2}}-\min \left(Z_{\mathrm{H}_{2}}, Z_{\mathrm{O}_{2}}\right) \\
Z_{\mathrm{O}_{2}, \mathrm{ex}} & =Z_{\mathrm{O}_{2}}-\min \left(Z_{\mathrm{H}_{2}}, Z_{\mathrm{O}_{2}}\right) \\
Z_{\mathrm{st}} & =2 \min \left(Z_{\mathrm{H}_{2}}, Z_{\mathrm{O}_{2}}\right)
\end{aligned}
$$

$Z_{\mathrm{H}_{2} \text {,ex }}$ and $Z_{\mathrm{O}_{2} \text {,ex }}$ represent the excess of the respective propellants from the stoichiometric proportion whereas $Z_{\mathrm{st}}$ is the fraction of both fresh propellants at stoichiometric proportion. One can verify that by definition $Z_{\mathrm{H}_{2}, \text { ex }}+Z_{\mathrm{O}_{2}, \text { ex }}+$ $Z_{\mathrm{st}}=Z_{\mathrm{H}_{2}}+Z_{\mathrm{O}_{2}} . Z_{\mathrm{st}}$ is reduced in case of fresh mixture dilution with burnt gases, which is also important for proper characterization of the fresh mixture quality. The results on averaged fields presented below are obtained by space and time averaging of instantaneous fields of $Z$-parameters following the same averaging procedure as for the density (simple arithmetic average).

\section{Results from the $3 \mathrm{D}$ simulation of the reactive flow}

A remark should be made concerning the RD propagation direction with respect to the orientation of the injection elements shown in Fig 7. Numerical tests have shown that the preferential direction of the RD propagation is defined by the orientation of the $\mathrm{O}_{2}$ jets of larger diameter, i.e. in the positive direction of the $x$-axis. In particular in the case $3 \mathrm{FS}$, the RD initially propagating in the opposite direction progressively degenerated and finally recovered the preferential direction. This particular case will not be discussed here in detail. All the 3D simulation results presented in the following sections are obtained for the $\mathrm{RD}$ propagation in the preferential direction. 


\subsection{Case 3CP: Injection of premixed propellants}

After flowfield initialisation in the 3D domain as it was explained in section 6. the simulation of Case 3CP took 180 ps of physical time necessary for full establishment of the flow in the chamber and then additional $60 \mu \mathrm{ss}$ (3 periods of RD) for data collection on the unsteady flowfield. Figure 9 shows the time history of average chamber pressure and total mass flow rate in the cross section separating the injector and chamber subdomains $(y=0.2 \mathrm{~mm})$ during the last 60 us. The total mass flow rate is normalized by the product of the nominal mass flux of $100 \mathrm{~kg} /\left(\mathrm{s} \cdot \mathrm{m}^{2}\right)$ and the cross section area. One can observe in this plot small fluctuations about the mean values of $0.202 \mathrm{MPa}$ for the chamber pressure and 1 for the normalised mass flow rate.

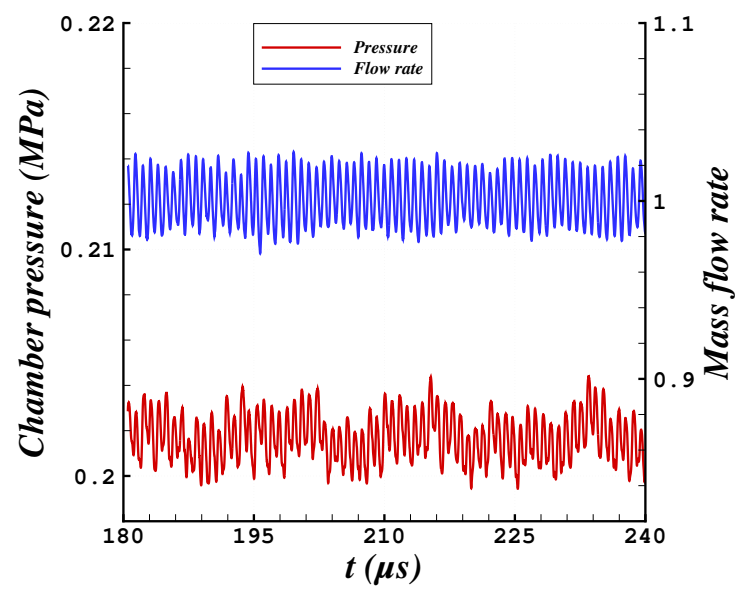

Figure 9: Time history of average chamber pressure and normalized total mass flow rate of Case $3 \mathrm{CP}$.

Time-averaged flowfields were obtained from two series of 60 snapshots every 1 ps and $15 \mathrm{~ns}$. It was found that both average flowfields were practically identical providing a proof that the flow is established in the chamber and that no bias is introduced by the choice of a sampling period as large as $1 \mu \mathrm{s}$. The average flowfields considered below are obtained with a sampling period of 1 us.

Figure 10 presents instantaneous fields at the median $z$-coordinate in comparison with averaged fields of the same flow parameters. One can see from this 


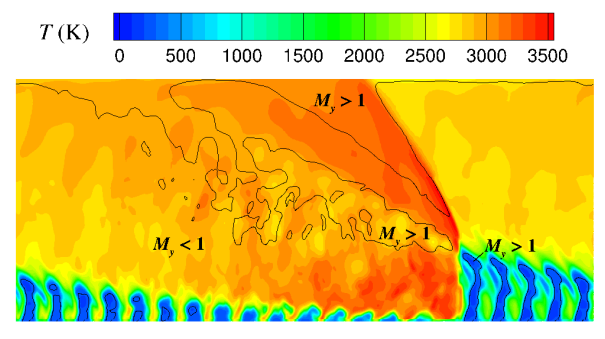

a)

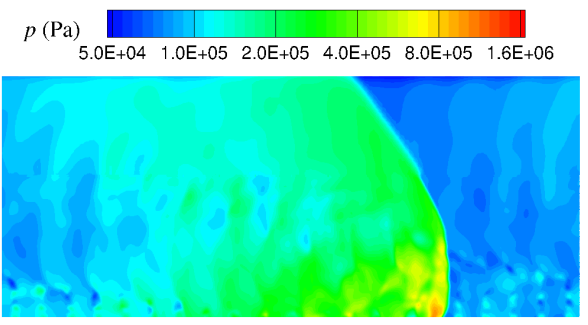

c)

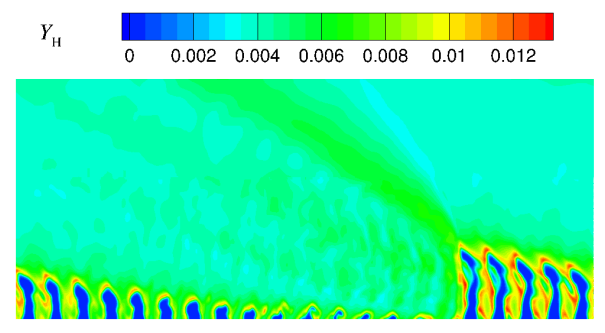

e)

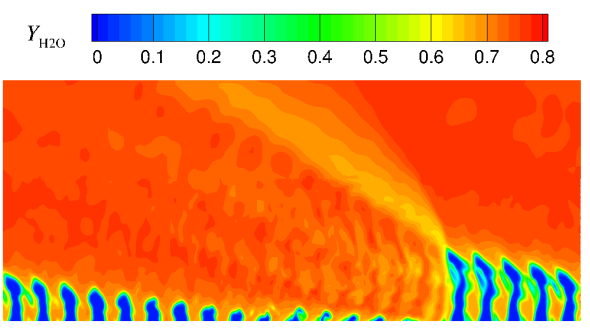

g)

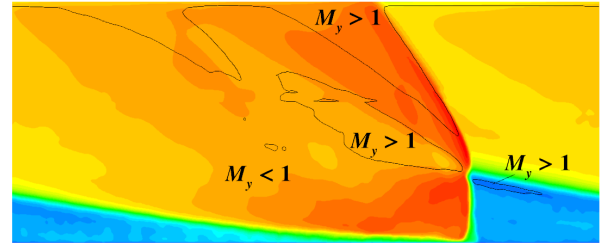

b)

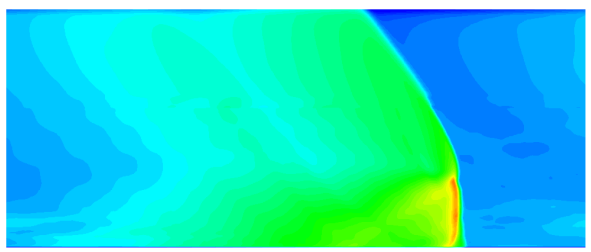

d)

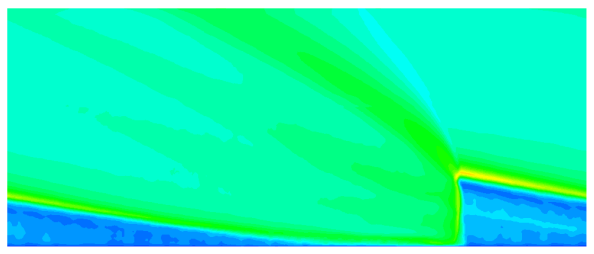

f)

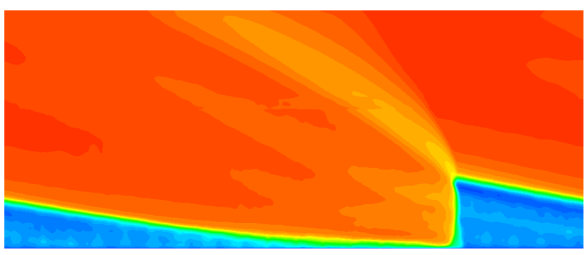

h)

Figure 10: Instantaneous (left) and averaged (right) flowfields for Case 3CP: a)-b) static temperature with the $M_{y}=1$ contour; c)-d) static pressure; e)-f) H-radical mass fraction; g)-h) $\mathrm{H}_{2} \mathrm{O}$ mass fraction. 
figure that the flow is unsteady and 3D, especially in the fresh mixture layer. The snapshots of temperature and species mass fractions show propellant jets surrounded with combustion products. Due to the heterogeneity of the fresh mixture layer, the detonation front is not smooth resulting in a perturbed flow behind it. As the detonation crosses a periodic pattern of propellant jets, it leads to the formation of acoustic waves with equidistant circular fronts, which are visible from the pressure snapshot. The acoustic perturbations generated by the previous detonation interact with the present one thus increasing the overall level of fluctuations. One can also see from the H-radical snapshot that deflagration fronts are formed around the premixed propellant jets immediately downstream of the injection plane so that the flame surface becomes very developed.

By comparing the flowfields of Cases $2 \mathrm{CP}$ (Fig 5 ) and 3CP, the following observations can be proposed. The overall features of the flow structure remain very similar if one considers the averaged flowfields of Case 3CP. In this latter case, the fresh mixture layer is thicker due to addition of combustion products, which is indicated by higher average temperature as well as non-zero mass fractions of $\mathrm{H}$ and $\mathrm{H}_{2} \mathrm{O}$. The detonation front is quite thin in the averaged flowfields hence its perturbations are rather small both in space and time. The detonation is however less intense than in Case 2CP providing significantly lower increase in pressure and temperature. The expansion and acceleration of combustion products in the chamber are also less important, which is indicated by higher pressure and temperature in front of the oblique shock as well as by the larger subsonic zone delimited by the $M_{y}=1$ contour. All these negative effects are due to the presence of combustion products, either remaining from the previous detonation or generated by deflagration, in the fresh mixture layer, which becomes less dense in terms of mass and chemical energy. By comparing the fields of $\mathrm{H}_{2} \mathrm{O}$ mass fraction, one may notice that behind the detonation, $\mathrm{H}_{2} \mathrm{O}$ is formed more gradually than in Case $2 \mathrm{CP}$; this is because radical recombination reactions are slower at lower pressure.

By using the method described in section 7.3 , the detonation propagation 

the averaged field of $\mathrm{H}$ radical as the maximum $y$ coordinate corresponding to the $H$ radical peak on the upper boundary of the fresh mixture layer, which gives $h=6.6 \mathrm{~mm}$. By considering a control volume as described in section 7.3 with $y_{\max }=h$, it is possible to determine the mass of fresh propellants $m_{\text {prop }}$ ${ }_{525}$ by integrating $\rho\left(Y_{\mathrm{H}_{2}}+Y_{\mathrm{O}_{2}}\right)$ where $Y_{\mathrm{H}_{2}}$ and $Y_{\mathrm{O}_{2}}$ are the mass fractions of $\mathrm{H}_{2}$ and $\mathrm{O}_{2}$ respectively. This mass is consumed by the detonation during a time period $\tau_{D}=l /\left(N_{\mathrm{inj}} V_{D}\right)$. Therefore, the mass flow rate burnt by the detonation is $\dot{m}_{\text {prop }} \approx m_{\text {prop }} / \tau_{D}$. It is found that $\dot{m}_{\text {prop }}$ is about $70 \%$ of the injected mass flow rate, which means that approximately $30 \%$ of propellant mass is consumed deflagration, with respect to $10 \%$ of Case $2 \mathrm{CP}$, is due to much more developed surface of flame front around the premixed propellant jets.

The following average conditions are determined within the control volume: static pressure $83.7 \mathrm{kPa}$; static temperature $563.4 \mathrm{~K}$; velocity $x$-component $\mathrm{O}_{2}$ mass fractions). Note that a part of the fresh mixture burnt by deflagration is transformed into hot gases above the fresh mixture layer, which are not contained in the control volume ; this explains the difference between the percentage of fresh mixture globally burnt by RD and present in the fresh layer upstream of RD. In comparison with the average conditions in the fresh mixture layer of Case 2CP, the pressure is higher by a factor of 1.33 (less important flow expansion after detonation) and the static temperature is higher by $325 \mathrm{~K}$ (presence of combustion products). One can also note that the $x$-component of velocity has different signs: it is negative in Case 2CP (see Fig 11 a) mainly because of a positive $x$-wise pressure gradient in the fresh mixture layer; in spite of the same factor also present in Case 3CP, the velocity sign is due to the orientation of the larger feeding tubes (see Fig,8), which creates an overall positive momentum of fresh mixture in the $x$ direction (see Fig $11 \mathrm{~b}$ ). An interesting fact is that, in spite of the heterogeneity of the fresh mixture layer, the detonation velocity with 
theoretical speed of Chapman-Jouguet detonation $D_{\mathrm{CJ}}=2681 \mathrm{~m} / \mathrm{s}$ determined for the average conditions in the chosen control volume.

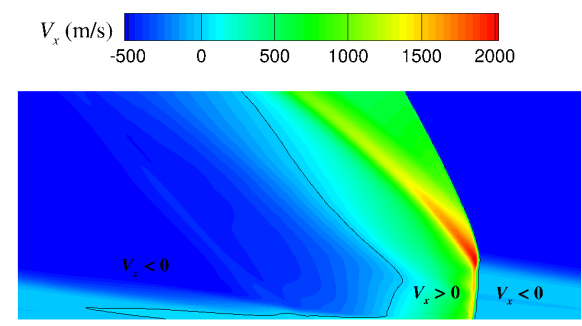

a)

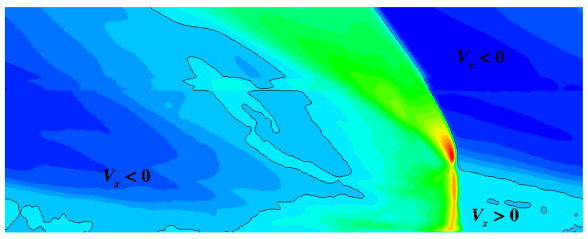

b)

Figure 11: Comparison of velocity $x$-component $V_{x}$ with the $V_{x}=0$ contour: a) Case 2CP; b) Case $3 \mathrm{CP}$, time-averaged.

\subsection{Cases 3CS and 3FS: Separate injection}

Only the results corresponding to an established flow in the chamber are

discussed here because the transitory regimes from the initial condition are not of particular interest. The averaged flowfields showed below were obtained from 60 snapshots with a sampling period of 1 ss. One can see from Tab 1 that Cases 3CS and 3FS are different only in terms of mesh resolution, whose effect on the simulation results need to be characterized. A brief analysis will be first made on this point before presenting the flowfield in detail. Figure 12 shows instantaneous fields in the $z$ mid-plane and averaged fields of static temperature for these two cases. By comparing the flowfields, one can see smaller turbulent structures in the instantaneous flowfield of Case 3FS due to the finer mesh resolution; on the other hand, the averaged fields have very similar topologies with minor differences of the temperature level in the corresponding zones.

The $M_{y}=1$ contour is plotted in these temperature fields and can be compared with the contours of Fig 5 a for Case $2 \mathrm{CP}$ and Fig $10 \mathrm{~b}$, b for Case $3 \mathrm{CP}$. The area of supersonic flow in the $y$ direction is further reduced in comparison to the previous cases. This observation means that the flow of combustion products is less accelerated during the expansion behind the detonation wave 
and, as a consequence, that combustion process is less efficient with the separate injection.

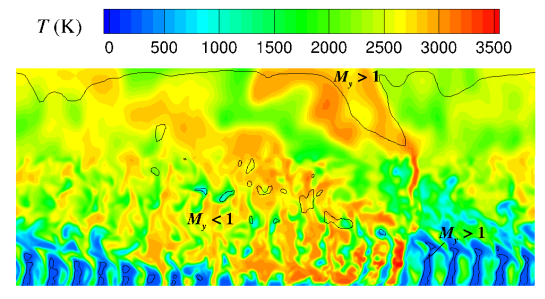

a)

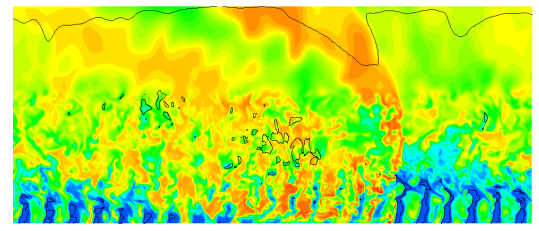

c)

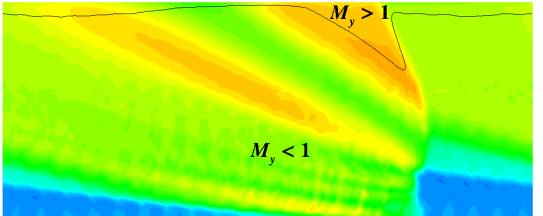

b)

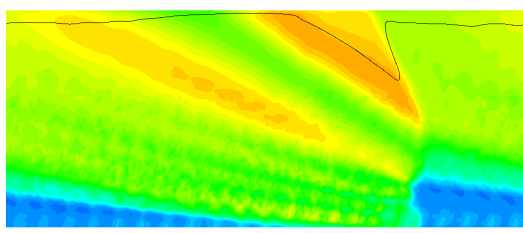

d)

Figure 12: Instantaneous (left) and averaged (right) fields of static temperature with the $M_{y}$ $=1$ contour: a)-b) Case 3CS; c)-d) Case 3FS.

Two important overall characteristics of the RD, the average static pressure on the injector wall and the detonation propagation speed, are compared in 575 Tab.2. They demonstrate that in both cases the RD has almost the same characteristics. It can be concluded from this comparative analysis of Cases 3CS and 3FS that the finer mesh resolution allows better representation of instantaneous flowfield with smaller turbulent structures, but has no important effect on the averaged flowfields as well as overall characteristics of the RD.

Table 2: Average static pressure on the injector wall $\bar{P}_{\mathrm{w}}$ and detonation propagation speed $V_{D}$ for Cases 3CS and 3FS.

\begin{tabular}{c|c|c} 
Case & $\overline{\boldsymbol{P}}_{\mathrm{w}}(\mathbf{M P a})$ & $\boldsymbol{V}_{\boldsymbol{D}}(\mathbf{m} / \mathbf{s})$ \\
\hline \hline 3CS & 0.1969 & 2166 \\
$3 \mathrm{FS}$ & 0.1972 & 2184
\end{tabular}




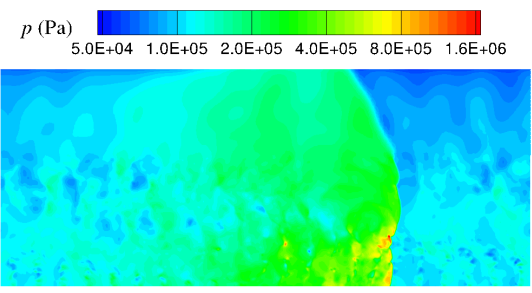

a)

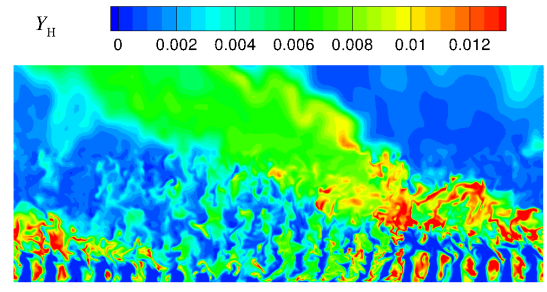

c)

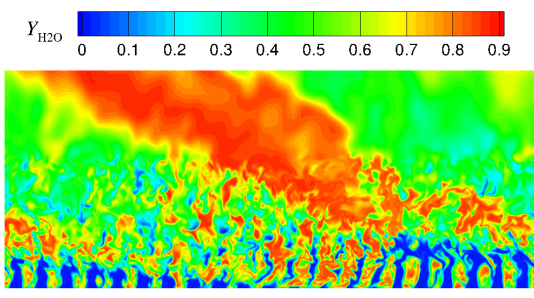

e)

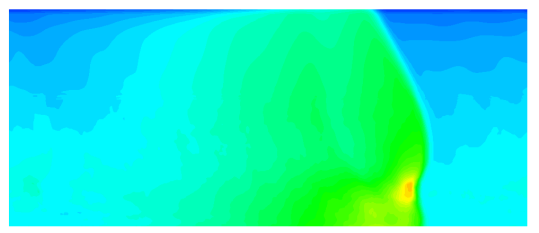

b)

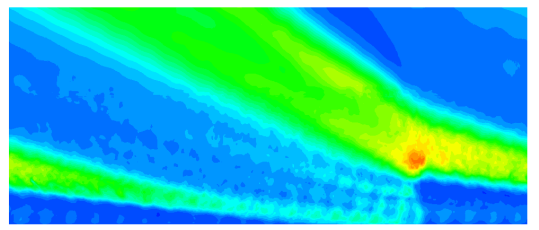

d)

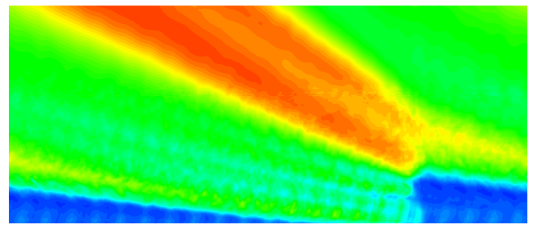

f)

Figure 13: Instantaneous (left) and averaged (right) flowfields of Case 3FS: a)-b) static pressure; c)-d) H-radical mass fraction; e)-f) $\mathrm{H}_{2} \mathrm{O}$ mass fraction.

To take advantage of finely resolved flowfield, the following results presented in this section will correspond to Case 3FS. In addition to the static temperature fields of Case 3FS in Fig 12, Fig 13 shows instantaneous fields of static pressure and mass fractions of $\mathrm{H}$ and $\mathrm{H}_{2} \mathrm{O}$ in the $z$ mid-plane together with averaged fields of the same quantities. In comparison with Case $3 \mathrm{CP}$, the instantaneous flow structure in the fresh mixture layer is chaotic with more perturbed boundaries of the propellant jets. The detonation front can be distinguished in the pressure fields; it is smeared in the averaged field indicating that the front shape is strongly perturbed both in space and time. Figure $13 \mathrm{~b}$ giving the averaged 
field of pressure evidences that the flow compression is not uniform along the detonation front with a zone of relatively high pressure close to the upper half of the fresh mixture layer where the propellant mixing is better than in the lower half. The overall pressure increase in the detonation wave is significantly reduced with respect to Case 3CP because of incomplete mixing of separately injected propellants. As a consequence of incomplete propellant mixing in the fresh mixture layer, many spots of relatively cold gas and unburnt propellants can be observed behind the detonation front in the snapshots of temperature and $\mathrm{H}_{2} \mathrm{O}$ fraction. The mixing process continues in the expanding gases after detonation, which is indicated by progressive dissipation of the cold spots. A more remarkable difference consists in intense combustion in a thick layer above the cold layer of fresh mixture, which is indicated by high levels of H-radical and $\mathrm{H}_{2} \mathrm{O}$ fractions before and after the detonation and shock waves.

The fields of $\mathrm{H}_{2} \mathrm{O}$ fraction in Fig 13 and $\mathrm{f}$ show a stratified repartition of this species within the burnt gas zone with distinct layers and strong overall variation in the vertical direction, which is very different from the corresponding fields of Cases 2CP and 3CP. To understand this behavior, profiles of averaged flow in a cross section at $x=25 \mathrm{~mm}$, which is far behind the detonation, are plotted in Fig.14. The total equivalence ratio $\phi_{t o t}$ is based on the balance of $\mathrm{H}$ and $\mathrm{O}$ atoms from all the species in the mixture. One can see from these profiles a strong overall variation of $\phi_{t o t}$ from the nominal level of 1 . The perturbations near the injector face are due to the propellant reinjection after the detonation passage. Let us consider the upper portion of the profiles above the point located at $y=1.7 \mathrm{~mm}$, for which $\phi_{t o t}=1$. There is a wide zone of fuel-lean combustion products $\left(0.33<\phi_{\text {tot }}<1\right)$ up to $y \approx 11.3 \mathrm{~mm}$. The three plots demonstrate similar trends within this lean zone. The temperature varies from a peak value of $2280 \mathrm{~K}$ to a minimum of $1645 \mathrm{~K}$ then goes up again to $2790 \mathrm{~K}$ whereas the $\mathrm{H}_{2} \mathrm{O}$ mass fraction decreases from 0.48 to 0.25 then goes up to 0.7 . The next zone $(y>11.3 \mathrm{~mm})$ is fuel-rich with a maximum equivalence ratio $\phi_{t o t} \approx 3.1$ at $y \approx 17.4 \mathrm{~mm}$. The temperature in this zone has a maximum of $2835 \mathrm{~K}$ at $y \approx 12 \mathrm{~mm}$ (slightly rich mixture) and a minimum of $2125 \mathrm{~K}$ whereas the $\mathrm{H}_{2} \mathrm{O}$ 

both minimums being at $y \approx 17.4 \mathrm{~mm}$.

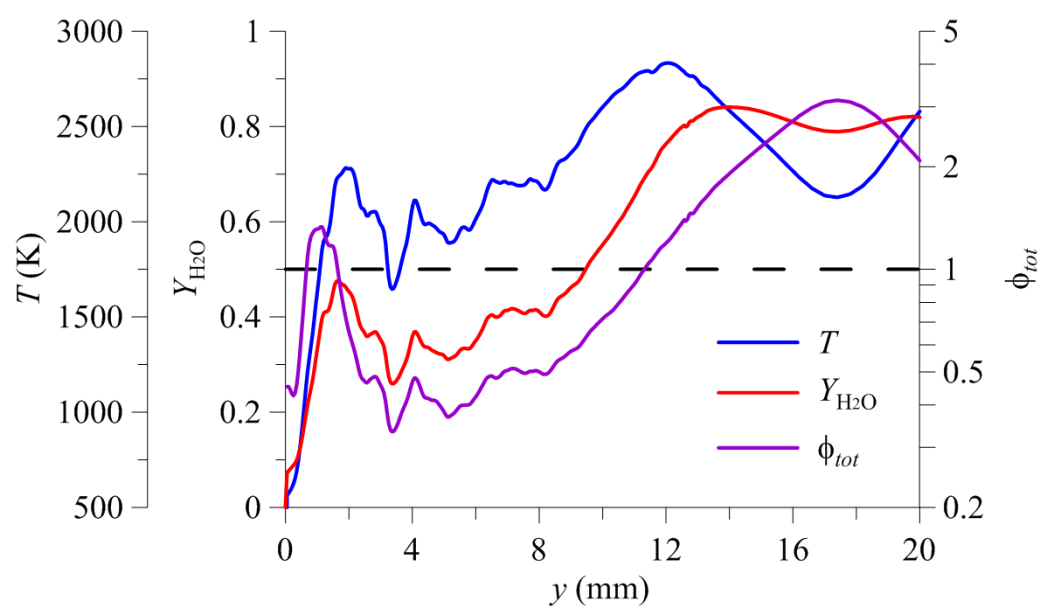

Figure 14: Profiles of averaged static temperature, $\mathrm{H}_{2} \mathrm{O}$ mass fraction and total equivalence ratio at $x=25 \mathrm{~mm}$.

Profiles of averaged $Z_{\mathrm{H}_{2} \text {,ex }}, Z_{\mathrm{O}_{2} \text {,ex }}, Z_{\mathrm{st}}$ and $Z_{\mathrm{BG}}$ at $x=25 \mathrm{~mm}$ are shown together with the corresponding profile of $\phi_{t o t}$ in Fig.15. Within the fuel-lean zone $(1.7 \mathrm{~mm}<y<11.3 \mathrm{~mm})$, the high level of $Z_{\mathrm{O}_{2} \text {,ex }}$ indicates a strong excess of $\mathrm{O}_{2}$ in the mixture; non-zero level of $Z_{\mathrm{H}_{2} \text {,ex }}$ points out the mixture heterogeneity because it means that fuel-rich pockets may exist even if in average there is a strong excess of $\mathrm{O}_{2}$. Non-zero values of $Z_{\text {st }}$ indicate incomplete combustion of cold gas pockets; $Z_{\mathrm{BG}}$ remains quite high within the interval [0.61;0.82]. Close to the point where $y=11.3 \mathrm{~mm}$ and $\phi_{t o t}=1$, maximums of $Z_{\mathrm{BG}}$ and $Z_{\mathrm{st}}$ are observed whereas both $Z_{\mathrm{H}_{2} \text {,ex }}$ and $Z_{\mathrm{O}_{2} \text {,ex }}$ are low. In the fuel-rich zone $(y>11.7$ $\mathrm{mm}$ ), there is a strong increase of $Z_{\mathrm{H}_{2} \text {,ex }}$ and reduction of $Z_{\mathrm{BG}}$ with extremums at the $\phi_{\text {tot }}$ maximum; both $Z_{\mathrm{O}_{2} \text {,ex }}$ and $Z_{\text {st }}$ vanish indicating almost complete dissipation of mixture heterogeneities.

Now with the help of the $Z$-parameters, the global repartition of mixture 635 components within the chamber will be analyzed in connection with the field of $\mathrm{H}_{2} \mathrm{O}$ mass fraction. Figure 16 shows the considered averaged fields, in which specific zones are delimited by black contour lines. The fresh mixture layer is 


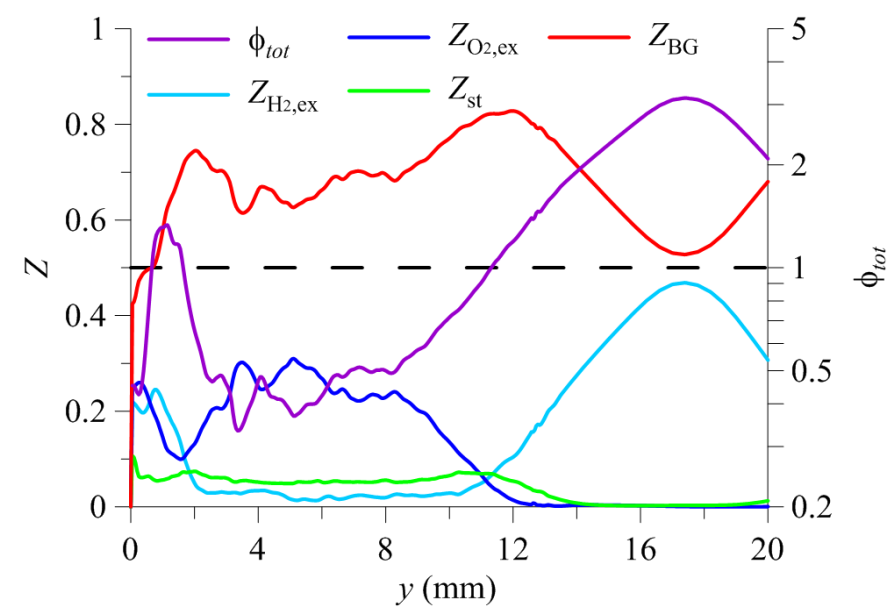

Figure 15: Profiles of averaged $Z$-parameters and total equivalence ratio at $x=25 \mathrm{~mm}$.

decomposed in three zones: the lower zone (1) is filled mainly with $\mathrm{O}_{2}$ and less with $\mathrm{H}_{2}$ and burnt gases; the intermediate zone (2) where the propellants are nearly stoichiometric but partially unmixed and diluted with burnt gases, which is indicated by $Z_{\text {st }}<1$; the upper zone (3) is fuel-rich and strongly diluted with burnt gases. Behind the detonation wave, there are three corresponding zones of combustion products: the oxidizer-rich zone (4) with a relatively low level of $\mathrm{H}_{2} \mathrm{O}$ mass fraction; the nearly stoichiometric zone (5), across which the $\mathrm{H}_{2} \mathrm{O}$ mass fraction rapidly increases; and the fuel-rich zone (6) with the highest level of $\mathrm{H}_{2} \mathrm{O}$ mass fraction. One can also identify these three zones in the profiles in Figs 14 and 15 . The last zone (7) represents a nearly stoichiometric layer between the fuel-rich fresh mixture of zone (3) and oxidizer-rich combustion products of zone (4) resulting from the previous detonation. As a particularity of zone (7), $\mathrm{H}_{2} \mathrm{O}$ is produced by combustion in a turbulent diffusion flame. The detonation propagation is driven by the most intense part of its front, which consumes mixed propellants from zones (1) and (2) and creates a zone of high pressure (9). The detonation is less intense near the injector face because of poor mixing and in zone (3) because of lack of oxidizer and strong dilution with burnt gases. The upper part of the detonation front (10) progressively 
degenerates and transforms into an oblique shock (11).

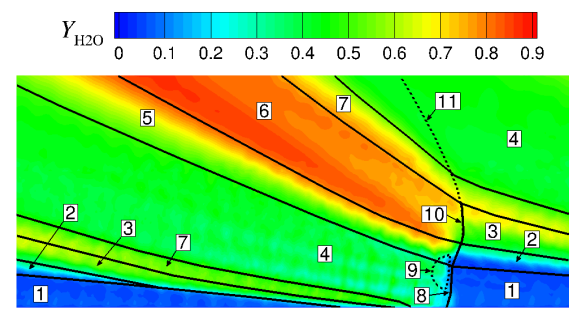

a)

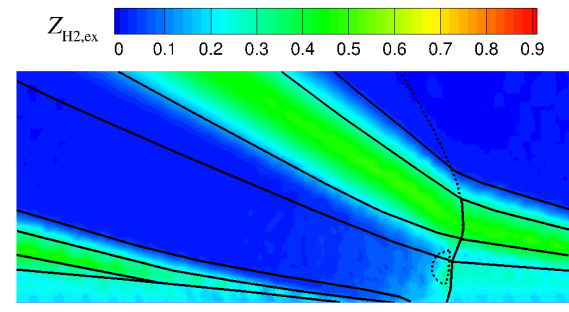

b)

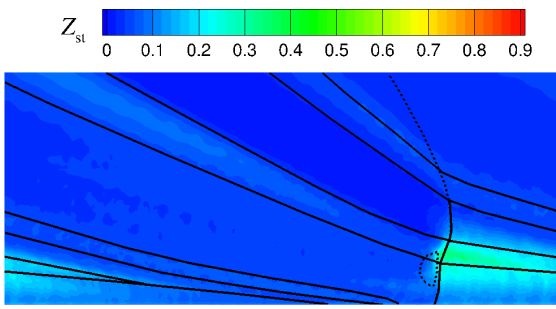

d)

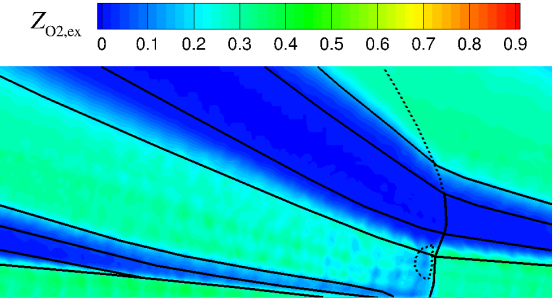

c)

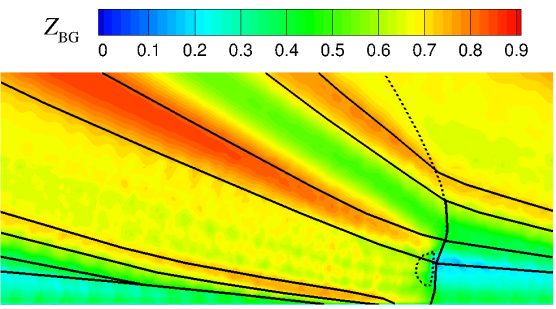

e)

Figure 16: Results of Case 3FS on averaged fields of mixture composition: a) $\mathrm{H}_{2} \mathrm{O}$ mass fraction; b) $Z_{\mathrm{H}_{2} \text {,ex }}$; c) $Z_{\mathrm{O}_{2} \text {,ex }}$; d) $Z_{\mathrm{st}}$; e) $Z_{\mathrm{BG}}$. Black contours define the following main zones: 1 oxidiser-rich fresh mixture; 2 nearly stoichiometric fresh mixture; 3 fuel-rich fresh mixture diluted with burnt gases; 4 oxidiser-rich combustion products from detonation; 5 nearly stoichiometric combustion products from detonation; 6 fuel-rich combustion products from detonation; 7 nearly stoichiometric combustion products from diffusion flame; 8 detonation front; 9 high-pressure zone behind the detonation front; 10 degenerating detonation front; 11 oblique shock.

It is now important to understand why the propellants are so strongly separated in the combustion chamber. By analyzing the evolution of propellant masses injected in the chamber on one period of $\mathrm{RD}$, it is found an excess of 

stoichiometric proportions for the following $12 \mu \mathrm{s}$, as indicated in Fig 17 by the points corresponding to $100 \%$ area of $\mathrm{O}_{2}$ orifices. Trying to eliminate this initial imbalance, simulations were also performed with $\mathrm{O}_{2}$ injection orifices whose area was $80 \%$ of the nominal value in order to increase the injection pressure of $\mathrm{O}_{2}$ (let us remind that the $\mathrm{O}_{2}$ flow rate is imposed at the entrance of the injection tube). The corresponding time evolutions of equivalence ratio from cumulated mass of injected propellants are plotted in Fig 17 indicating that injected masses are nearly stoichiometric all the time with the reduced $\mathrm{O}_{2}$ orifices. However in spite of this improvement, the obtained results on the chamber flowfield did not show any significant change in the overall repartition of the main mixture components.

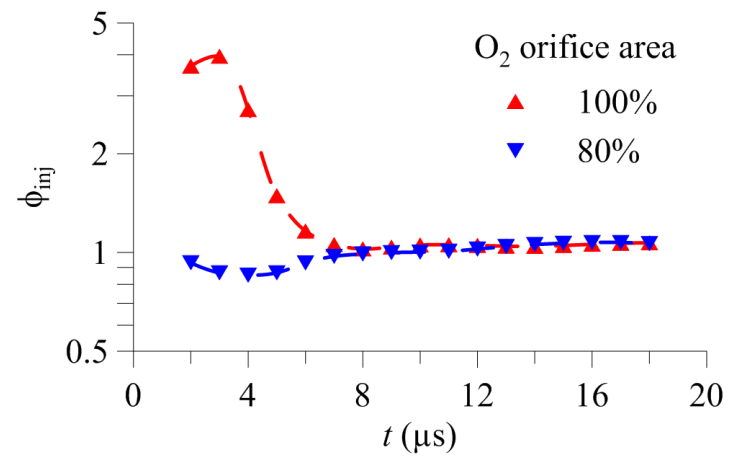

Figure 17: Equivalence ratio from cumulated mass of injected propellants during one time period of RD for the nominal and reduced area of $\mathrm{O}_{2}$ injection orifices.

The real cause of the observed separation of propellants in the fresh mixture layer is due to different velocities of propellant jets. At the same injection Mach number, the $\mathrm{H}_{2}$ jets have much higher initial velocity than the $\mathrm{O}_{2}$ jets. As the injected propellants cannot instantly mix, $\mathrm{O}_{2}$ is mostly accumulated close to the injector face and forms the oxidizer-rich zone of the fresh mixture layer. In spite of the low density, the $\mathrm{H}_{2}$ jets preserve their high velocity and penetrate deeper into the burnt gases from the previous detonation, thus forming the fuel-rich zone of the fresh mixture layer. 
In order to characterize the fresh mixture layer in front of the detonation, a control volume is considered as described in section 7.3. It is not possible to determine the height of the fresh mixture layer from the flowfields because it has no distinct upper boundary. It was decided to take $y_{\max }$ at the distance at which the mass contained in the control volume is equal to the total propellant mass $m_{\text {tot }}$ injected during a time period $\tau_{D}=l /\left(N_{\mathrm{inj}} V_{D}\right)$. The obtained values of $y_{\max }$ for Cases 3CS and 3FS are given in Tab 3 together with the mass percentage of fresh propellants mixed at stoichiometric proportions and of burnt gases. The results are close for both cases except $m_{\mathrm{st}} / m_{t o t}$, for which the value for Case 3FS is lower because of reduced numerical diffusion due to finer mesh resolution. These estimations show that not more than $18 \%$ of the injected propellant mass is mixed and can be burnt by the detonation (the remaining part is however burnt by deflagration); nearly $70 \%$ of this mass remain unmixed due to the short time period between successive detonations and the effect of propellant separate injection; almost $15 \%$ of remaining mass is represented by burnt gases, which can be either residual from the previous detonation or generated by combustion of mixed fresh propellants.

Table 3: Height of the control volume, mass percentage of mixed propellants and burnt gases in the fresh mixture layer in front of the detonation for Cases 3CS and 3FS.

\begin{tabular}{c|c|c|c} 
Case & $\boldsymbol{y}_{\text {max }}(\mathbf{m m})$ & $\boldsymbol{m}_{\mathbf{s t}} / \boldsymbol{m}_{\boldsymbol{t o t}}$ & $\boldsymbol{m}_{\mathbf{B G}} / \boldsymbol{m}_{\boldsymbol{t o t}}$ \\
\hline \hline 3CS & 8.1 & $18.2 \%$ & $14.8 \%$ \\
$3 \mathrm{FS}$ & 8.2 & $16.5 \%$ & $14.5 \%$
\end{tabular}

Average conditions in the fresh mixture layer are not determined for Cases 3CS and 3FS because they are not representative due to strong heterogeneity and propellant separation within the fresh mixture layer.

\subsection{Discussion on the simulated cases}

The main results from all the simulated cases are summarised in Tab 4 The results obtained for the 3D cases will be compared to the most idealistic Case $2 \mathrm{CP}$. The detonation propagation speed is by $5.6 \%$ higher for Case $3 \mathrm{CP}$ 
because of the effect of hot gases in the fresh mixture layer; it is however by approximately 19\% lower for Cases 3CS and 3FS because of poor quality of fresh mixture due to incomplete mixing and propellant separation effect. The detonation front height is larger by $35 \%$ for Case $3 \mathrm{CP}$ because of burnt gases in the fresh mixture layer. For Cases $3 \mathrm{CS}$ and $3 \mathrm{FS}, h$ is defined equal to the control volume height $y_{\max }$. The overall mixing efficiency $\eta_{\operatorname{mix}}$ is unity for Cases $2 \mathrm{CP}$ and $3 \mathrm{CP}$ with premixed injection; for Cases 3CS and 3FS, the highest estimation $\eta_{\text {mix }}$ is $\left(m_{\mathrm{st}}+m_{\mathrm{BG}}\right) / m_{\text {tot }} \approx 33 \%$ by assuming that burnt gases in the fresh mixture layer are produced by combustion of mixed propellants and the lowest estimation $\eta_{\text {mix }}$ is $m_{\text {st }} / m_{\text {tot }} \approx 18 \%$ by assuming that there are only residual burnt gases from the previous detonation. The efficiency of combustion 715 by detonation $\eta_{D}$ is defined as a fraction of the injected propellant mass burnt by detonation; for Cases $2 \mathrm{CP}$ and $3 \mathrm{CP}$, it is determined from the total mass of fresh propellants in the control volume in front of the detonation whereas for Cases $3 \mathrm{CS}$ and $3 \mathrm{FS}, \eta_{D}=m_{\mathrm{st}} / m_{\text {tot }}$ because only mixed propellants can be burnt by detonation. The last parameter is the average pressure on the injector wall $\bar{P}_{\mathrm{w}}$, which is also an indicator of RD efficiency at the same mass flow rate. In spite of the higher detonation speed of Case $3 \mathrm{CP}, \bar{P}_{\mathrm{w}}$ is lower by $15 \%$ because of excessive consumption of premixed propellants by deflagration and the effect of hot gases in the fresh mixture reducing the detonation intensity. In Cases $3 \mathrm{CS}$ and $3 \mathrm{FS}$, the poor mixing results in a reduced detonation speed. However ${ }_{725} \bar{P}_{\mathrm{w}}$ is lower only by $17 \%$, which is not so different from the result of Case $3 \mathrm{CP}$.

Table 4: Detonation propagation speed, detonation front height, mixing efficiency, efficiency of combustion by detonation, average pressure on the injector wall for the simulated cases.

\begin{tabular}{c|c|c|c|c|c} 
Case & $\boldsymbol{V}_{\boldsymbol{D}}(\mathbf{m} / \mathbf{s})$ & $\boldsymbol{h}(\mathbf{m m})$ & $\boldsymbol{\eta}_{\mathbf{m i x}}$ & $\boldsymbol{\eta}_{\boldsymbol{D}}$ & $\overline{\boldsymbol{P}}_{\mathbf{w}}(\mathbf{M P a})$ \\
\hline \hline 2CP & 2679 & 4.9 & 1 & 0.9 & 0.2376 \\
$3 \mathrm{CP}$ & 2828 & 6.6 & 1 & 0.72 & 0.2017 \\
$3 \mathrm{CS}$ & 2166 & 8.1 & 0.182 to 0.33 & 0.182 & 0.1969 \\
$3 \mathrm{FS}$ & 2184 & 8.2 & 0.165 to 0.31 & 0.165 & 0.1975
\end{tabular}


The results on $\bar{P}_{\mathrm{w}}$ become clear from the comparison of the pressure profiles on the injector wall deduced of the averaged flowfields as shown in Fig 18 . The pressure profile of Case $3 \mathrm{CP}$ has a $50 \%$ lower peak with a quick drop behind the detonation followed by a slight increase on the interval $0.6<x / l<0.73$ and a further smooth decrease on $x / l<0.6$; the observed pressure increase after the main peak is due to transverse shocks, which are caused by detonation front perturbations when the detonation crosses heterogeneities in the fresh mixture layer. The profiles of Cases 3CS and 3FS are almost coincident without any sharp peak because, on the one hand, the zone of highest pressure is situated at a certain distance from the wall and, on the other hand, the pressure peak is smeared by shock front perturbations; the pressure decrease is quite gradual behind the detonation probably due to distributed heat release from the burning gas pockets.

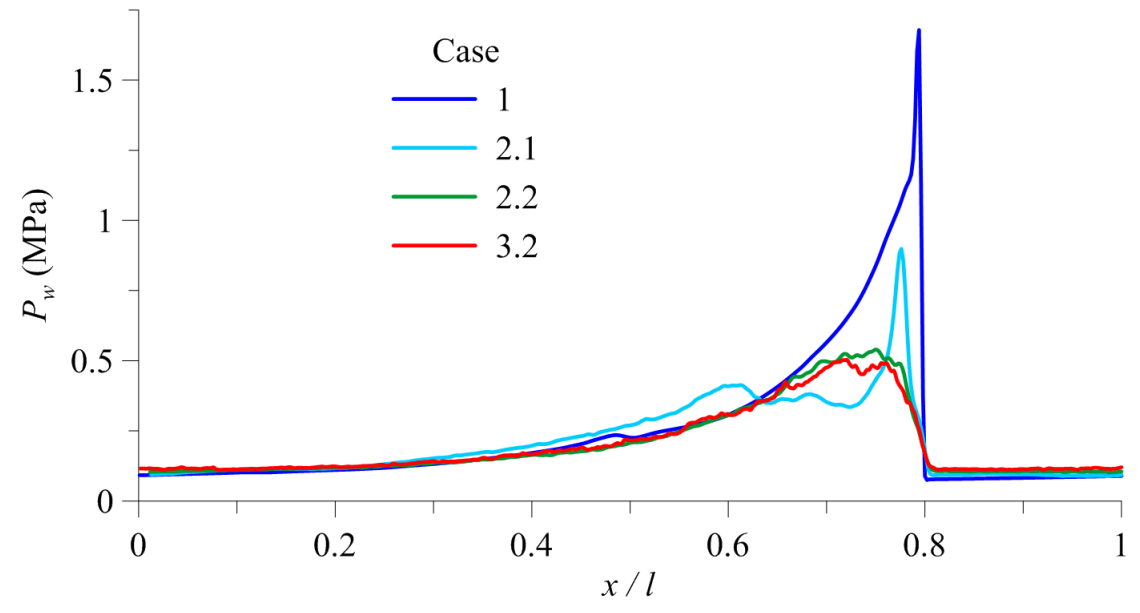

Figure 18: Pressure profiles on the injector wall from the averaged flowfields.

\section{Conclusion}

A numerical study has been carried out providing an important set of simulation results on the stable propagation of a $\mathrm{RD}$ fed with the $\mathrm{H}_{2}$ and $\mathrm{O}_{2}$ gaseous propellants. Three different injection regimes have been analyzed with 
increasing complexity of the propellant injection and mixing processes from an idealistic one with premixed uniformly distributed injection to the most realistic one with separate injection through discrete holes. The simulation results for the different injection regimes have been systematically compared in order to provide physical interpretation of the observed changes related to the $\mathrm{RD}$ propagation conditions and efficiency of the combustion process.

The 3D simulation results have been analyzed by considering instantaneous and averaged flowfields. Specific methods have been used to evaluate the RD propagation speed, average conditions in the fresh mixture layer and efficiency of the mixing and combustion processes. Special parameters characterizing the mixture composition containing fresh propellants and burnt gases have been introduced for proper visualization of the composition field and correct assessment of the mixture quality.

By simulating the established RD propagation over a series of injection elements, previously designed for separate injection of gaseous $\mathrm{H}_{2}$ and $\mathrm{O}_{2}$ at stoichiometric proportions, with periodic conditions on the lateral boundaries, it was possible to study the dynamic behavior of the injector as well as the formation of the fresh mixture layer between two consecutive passages of the RD. Several important findings have been made by analyzing the simulation results.

The use of discrete holes for propellant injection creates conditions for fresh mixture dilution with hot gases, which can either remain from the previous $\mathrm{RD}$ passage or be produced by combustion of the injected propellants. In the case of premixed injection through discrete holes, nearly $30 \%$ of the injected mass is consumed by deflagrative combustion because of a developed flame surface around the propellant jets. The dilution with hot gases results in considerable reduction of the pressure rise across the detonation wave and overall pressure decrease on the injection wall but not necessarily in a decrease of the RD propagation speed, which remains high due to the hot fresh mixture. In spite of the heterogeneities within the fresh mixture layer, the RD propagation speed differs by only a few percent from the CJ detonation speed determined for the fresh mixture average conditions in front of the RD. 
With the separate propellant injection, the main negative factor is the pro-

pellant stratification due to different velocities of the $\mathrm{H}_{2}$ and $\mathrm{O}_{2}$ jets, which leads to the formation of an oxygen-rich zone near the injection wall and a hydrogen-rich zone in the upper part of the fresh mixture layer. This stratification results in poor mixing efficiency (between 17\% and 33\%) within the fresh mixture layer and, as a consequence, in less than $20 \%$ of fresh propellants burnt by detonation as well as globally incomplete combustion. The identification and explication of the propellant stratification effect is a very important finding of this study, which needs to be considered when evaluating the injection element design. This critical effect could not be found in our previous study [30] devoted to the mixing simulation under conditions of established injection because it was not possible to take into account the dynamics of the refill process as well as the presence of burnt gases.

The simulation of the injector operation under conditions of RD propagation is essential for a proper evaluation of the injector design. However, such a simulation is quite expensive for an optimization process requiring a relatively large number of calculations. One of the further steps to enhance the design methodology could be the accurate modeling of the transitory refill process with a single injection element under conditions of expanding burnt gases produced by the RD. This technique would give us the possibility to optimize the injector design at affordable cost. In addition, it would be possible to use a better mesh resolution and an adapted modeling approach for the deflagration on the contact surface between the fresh propellants and burnt gases. The RD simulation with multiple injection elements would be used for the final validation step only.

\section{References}

[1] B. V. Voitsekhovskii, Stationary spin detonation, Soviet Journal of Applied Mechanics and Technical Physics 3 (1960) 157-164.

[2] J. A. Nicholls, R. E. Cullen, The feasability of a rotating detonation wave rocket motor, Tech. rep., RPL-DTR-64-113, University of Michigan (1964). 
[3] T. Adamson, G. Olsson, Perfomance analysis of a rotating detonation wave rocket engine, Acta Astronautica 13 (4) (1967) 405-415.

[4] P. Wolanski, Detonative propulsion, Proceedings of the Combustion Institute 34 (2013) 125-158.

[5] F. K. Lu, E. M. Braun, Rotating detonation wave propulsion: Experimental challenges, modeling, and engine concepts, Journal of Propulsion and Power 30 (5) (2014) 1125-1142.

[6] Z. Rui, W. Dan, W. Jianping, Progress of continuously rotating detonation engines, Chinese Journal of Aeronautics 29 (1) (2016) 15-29.

[7] F. A. Bykovskii, S. A. Zhdan, E. F. Vedernikov, Continuous spin detonations, Journal of Propulsion and Power 22 (6) (2006) 1204-1216.

[8] F. A. Bykovskii, S. A. Zhdan, E. F. Vedernikov, Continuous spin detonation of hydrogen-oxygen mixtures. 1. annular cylindrical combustors, Combustion, Explosion, and Shock Waves 44 (2) (2008) 150-162.

[9] F. A. Bykovskii, S. A. Zhdan, E. F. Vedernikov, Continuous spin detonation of synthesis gas-air mixtures, Combustion, Explosion, and Shock Waves 49 (4) (2013) 435-441.

[10] J. Kindracki, P. Wolanski, Z. Gut, Experimental research on the rotating detonation in gaseous fuels-oxygen mixtures, Shock Waves 21 (2011) 75-84.

[11] J. Kindracki, Experimental research on the rotating detonation in liquid fuel-gaseous air mixtures, Aerospace Science and Technology 43 (2015) 445453.

[12] B. Le Naour, F. Falempin, M. Flore, Recent experimental results obtained on continuous detonation wave engine, 17th AIAA International Space Planes and Hypersonic Systems and Technologies Conference, (AIAA-20112235). 
[13] E. M. Braun, F. K. Lu, D. R. Wilson, J. A. Camberos, Airbreathing rotating detonation wave engine cycle analysis, Aerospace Science and Technology 27 (2013) 201-208.

[14] D. M. Davidenko, Y. Eude, I. Gökalp, F. Falempin, Theoretical and numerical studies on continuous detonation wave engines, 17th AIAA International Space Planes and Hypersonic Systems and Technologies Conference, (AIAA-2011-2334).

[15] S. A. Zhdan, F. A. Bykovskii, E. F. Vedernikov, Mathematical model of a rotating detonation wave in a hydrogen-oxygen mixture, Combustion, Explosion, and Shock Waves 43 (4) (2007) 449-459.

[16] D. Schwer, K. Kailasanath, Numerical investigation of the physics of rotating-detonation-engines, Proceedings of the Combustion Institute 33 (2010) 2195-2202.

[17] D. Schwer, K. Kailasanath, Fluid dynamics of rotating detonation engines with hydrogen and hydrocarbon fuels, Proceedings of the Combustion Institute 34 (2013) 1991-1998.

[18] T.-H. Yi, J. Lou, C. Turangan, J.-Y. Choi, P. Wolanski, Propulsive performance of a continuously rotating detonation engine, Journal of Propulsion and Power 27 (1) (2011) 171-181.

[19] Z. Pan, B. Fan, X. Zhang, M. Gui, G. Dong, Wavelet pattern and selfsustained mechanism of gaseous detonation rotating in a coaxial cylinder, Combustion and Flame 158 (2011) 2220-2228.

[20] Y. Uemura, A. K. Hayashi, M. Asahara, N. Tsuboi, E. Yamada, Transverse wave generation mechanism in rotating detonation, Proceedings of the Combustion Institute 34 (2013) 1981-1989.

[21] S.-J. Liu, Z.-Y. Lin, W.-D. Liu, W. Lin, M.-B. Sun, Experimental and three-dimensional numerical investigations on h2/air continuous rotating 
detonation wave, Proceedings of the Institution of Mechanical Engineers, Part G: Journal of Aerospace 227 (2013) 326-341.

[22] D. M. Davidenko, I. Gökalp, A. N. Kudryavtsev, Numerical simulation of a continuous detonation in a layer of hydrogen-oxygen mixture with periodic conditions, G.D. Roy and S.M. Frolov (Eds.), Deflagrative and detonative combustion (pp.407-422). Moscow: Torus Press (2010), ISBN 978-5-94588071-9.

[23] Y. Eude, D. M. Davidenko, I. Gökalp, F. Falempin, Numerical simulation and analysis of a $3 \mathrm{~d}$ continuous detonation under rocket engine conditions, 4th European Conference for Aerospace Sciences (EUCASS 2011), 4-8 July. Saint Petersburg, Russia.

[24] M. Liu, R. Zhou, J.-P. Wang, Numerical investigation of different injection patterns in rotating detonation engines, Combustion, Science and Technology 187 (2015) 343-361.

[25] D. Schwer, K. Kailasanath, Feedback into mixture plenums in rotating detonation engines, 50th AIAA Aerospace Sciences Meeting including the New Horizons Forum and Aerospace Exposition, (AIAA-2012-0617).

[26] S. M. Frolov, A. V. Dubrovskii, V. S. Ivanov, Three-dimensional numerical simulation of the operation of a rotating-detonation chamber with separate supply of fuel and oxidizer, Russian Journal of Physical Chemistry B 7 (1) (2013) 35-43.

[27] D. A. Schwer, K. Kailasanath, Towards non-premixed injection modeling of rotating detonation engines, 51st AIAA/SAE/ASEE Joint Propulsion Conference, AIAA Propulsion and Energy Forum, (AIAA-2015-3782).

[28] R. Driscoll, A. St. George, E. J. Gutmark, Numerical investigation of injection within an axisymmetric rotating detonation engine, International Journal of Hydrogen Energy 41 (2016) 2052-2063. 
[29] P. A. T. Cocks, A. T. Holley, B. A. Rankin, High fidelity simulations of a non-premixed rotating detonation engine, 54th AIAA Aerospace Sciences Meeting, AIAA SciTech Forum, (AIAA 2016-0125).

[30] T. Gaillard, D. Davidenko, F. Dupoirieux, Numerical optimisation in non reacting conditions of the injector geometry for a continuous detonation wave rocket engine, Acta Astronautica 111 (2015) 334-444.

[31] Y. Eude, D. M. Davidenko, I. Gökalp, F. Falempin, Use of the adaptive mesh refinement for 3d simulations of a CDWRE (Continuous Detonation Wave Rocket Engine), 17th AIAA International Space Planes and Hypersonic Systems and Technologies Conference, (AIAA-2011-2236).

[32] A. Refloch et al., Cedre software, Aerospace Lab Journal 2. URL http://www . aerospacelab-journal .org/CEDRE-Sof tware

${ }_{895}$ [33] D. Scherrer et al., Recent cedre applications, Aerospace Lab Journal 2. URL http://www . aerospacelab-journal.org/node/101

[34] Chemical-kinetic mechanisms for combustion applications, San Diego Mechanism web page, Mechanical and Aerospace Engineering (Combustion Research), University of California at San Diego. URL http://combustion.ucsd.edu

[35] A. L. Sánchez, F. A. Williams, Recent advances in understanding of flammability characteristics of hydrogen, Progress in Energy and Combustion Science, Vol. 41, 2014, pp. 1-55. 